\title{
SUPPLEMENT TO "CAUSAL GRAPHICAL MODELS IN SYSTEMS GENETICS: A UNIFIED FRAMEWORK FOR JOINT INFERENCE OF CAUSAL NETWORK AND GENETIC ARCHITECTURE FOR CORRELATED PHENOTYPES"
}

\author{
By Elias Chaibub Neto, Mark P. Keller, Alan D. Attie, and Brian S. Yandell
}

\author{
University of Wisconsin-Madison
}

1. The QTLnet algorithm. In this section we present a Metropolis-Hastings algorithm that at each step proposes a slightly modified phenotype network and fits the genetic architecture conditional on this proposed network. The Metropolis-Hastings step involves proposing a new network with a single modification from the last accepted network. Allowed modifications include single arrow deletions, additions or reversions that do not result in a cyclic network. (See Figure S1 for details of the mechanics for the proposal scheme and for the computation of the proposal probabilities.) The Metropolis-Hastings proposal ratio $q\left(\mathcal{M}_{\text {old }} \mid \mathcal{M}_{\text {new }}\right) / q\left(\mathcal{M}_{\text {new }} \mid \mathcal{M}_{\text {old }}\right)$ is the ratio of the neighborhood sizes of the two networks involved in the proposal modification. The neighborhood of a DAG, ne $(\mathcal{M})$ is defined as the number of DAGs that can be obtained from the present DAG in a single proposal modification. Specifically, the QTLnet algorithm proceeds as follows:

1. Given a phenotype network structure $\mathcal{M}_{\text {old }}$, propose a new network, $\mathcal{M}_{\text {new }}$, with a proposal probability $q\left(\mathcal{M}_{\text {new }} \mid \mathcal{M}_{\text {old }}\right)=1 /\left|n e\left(\mathcal{M}_{\text {old }}\right)\right|, \mathcal{M}_{\text {new }} \in n e\left(\mathcal{M}_{\text {old }}\right)$.

2. Factor the likelihood according to $\mathcal{M}_{\text {new }}$ and perform mapping analysis of $y_{t}$ conditional on its parents, $p a\left(y_{t}\right)$, for each phenotype $y_{t}$ separately.

3. Estimate each piece of the marginal likelihood, $p\left(y_{t} \mid \mathbf{q}_{t}, p a\left(y_{t}\right)\right)$, and combine then to obtain $\hat{p}\left(\mathbf{y} \mid \mathbf{q}, \mathcal{M}_{\text {new }}\right)$.

4. Accept $\mathcal{M}_{\text {new }}$ with probability

$$
\begin{aligned}
\alpha & =\min \left\{1, \frac{p\left(\mathcal{M}_{\text {new }} \mid \mathbf{y}, \mathbf{q}\right)}{p\left(\mathcal{M}_{\text {old }} \mid \mathbf{y}, \mathbf{q}\right)} \frac{q\left(\mathcal{M}_{\text {old }} \mid \mathcal{M}_{\text {new }}\right)}{q\left(\mathcal{M}_{\text {new }} \mid \mathcal{M}_{\text {old }}\right)}\right\} \\
& =\min \left\{1, \frac{\hat{p}\left(\mathbf{y} \mid \mathbf{q}, \mathcal{M}_{\text {new }}\right) p\left(\mathcal{M}_{\text {new }}\right)}{\hat{p}\left(\mathbf{y} \mid \mathbf{q}, \mathcal{M}_{\text {old }}\right) p\left(\mathcal{M}_{\text {old }}\right)} \frac{q\left(\mathcal{M}_{\text {old }} \mid \mathcal{M}_{\text {new }}\right)}{q\left(\mathcal{M}_{\text {new }} \mid \mathcal{M}_{\text {old }}\right)}\right\} .
\end{aligned}
$$

Mapping analysis and the estimation of each component, $p\left(y_{t} \mid \mathbf{q}_{t}, p a\left(y_{t}\right)\right)$, of the marginal likelihood can be done in two different ways: (1) perform Bayesian interval mapping (Yi et al. 2005 ) and use the posterior samples generated in the mapping analysis to estimate the marginal likelihood pieces using the stabilized harmonic mean estimator (Raftery et al 2007); (2) perform classical interval mapping, sum the BIC score for each piece of the sampling model to obtain the BIC score of the whole network, and then use the asymptotic approximation of the Bayes factors by BIC scores. It is a well known ( $c f$. Kass and Raftery 1995) that when sample size is big enough, we can approximate the Bayes factor comparing old and new models by, in this case,

$$
\frac{p\left(\mathbf{y} \mid \mathbf{q}, \mathcal{M}_{\text {new }}\right)}{p\left(\mathbf{y} \mid \mathbf{q}, \mathcal{M}_{\text {old }}\right)} \approx \exp \left\{-\frac{1}{2}\left(B I C_{\mathcal{M}_{\text {new }}}-B I C_{\mathcal{M}_{\text {old }}}\right)\right\}
$$


Step 2 in the QTLnet algorithm is the most computationally demanding. Given the big size of the graph space even for small networks, efficient computation is key. The first obvious gain in efficiency is to store the estimates of the $p\left(y_{t} \mid \mathbf{q}_{t}, p a\left(y_{t}\right)\right)$ as we proceed. Because $\mathcal{M}_{\text {new }}$ will only slightly differ from $\mathcal{M}_{\text {old }}$, most of the factor pieces of the network will not need to be recomputed. Actually, we will only need to perform new mapping analysis to the pair of nodes involved in an arrow reversal or to the child node involved in an arrow addition or deletion. In the first case, we need to redo the mapping analysis for both nodes when we reverse the arrow $y_{1} \rightarrow y_{2}$ to $y_{1} \leftarrow y_{2}$, since we need to include $y_{2}$ as a covariate in the mapping of $y_{1}$ and we need to exclude $y_{1}$ as a covariate in the mapping of $y_{2}$. For a child node, when we add (or delete) an arrow from $y_{1}$ to $y_{2}$ we need to include (or exclude) $y_{1}$ as a covariate in the mapping of $y_{2}$.

Another way to improve computational efficiency is to first perform a multiple-trait multipleQTL analysis to all phenotypes with the seemingly unrelated regression model (Banerjee et al 2008) and then only consider the QTLs detected by this analysis in the rest of the analysis. In this way, instead of considering all locations in the genome as potential QTLs every time we need to redo a mapping analysis, we simply consider the locations that were detected by the seemingly unrelated regression analysis, greatly decreasing the total amount of necessary computations. Still another way to decrease computational time, in Step 2, is to perform classical interval mapping instead of Bayesian interval mapping, and then use the asymptotic approximation of the Bayes factors by BIC scores. We should point out, however, that since classical interval mapping is less powered to detect multiple QTL and misses epistasis altogether, the gain in computational efficiency provided by this approach comes at the expense of performing an inferior job in terms of genetic architecture inference.

2. Supplementary tables. Here we present supplementary tables for the simulation study and the real data example.

\begin{tabular}{l|cccc|cccc|c}
\hline & \multicolumn{4}{|c|}{ strong signal } & \multicolumn{4}{c|}{ weak signal } & Expected \\
Phenotypes & $Q_{1}$ & $Q_{2}$ & $Q_{4}$ & $Q_{5}$ & $Q_{1}$ & $Q_{2}$ & $Q_{4}$ & $Q_{5}$ & architecture \\
\hline$Y_{1}$ & 0.997 & 0.000 & 0.000 & 0.000 & 0.431 & 0.000 & 0.000 & 0.000 & $\left\{Q_{1}\right\}$ \\
$Y_{1} \mid Y_{2}$ & 0.969 & 0.948 & 0.000 & 0.000 & 0.405 & 0.006 & 0.000 & 0.000 & $\left\{Q_{1}, Q_{2}\right\}$ \\
$Y_{1} \mid Y_{3}$ & 0.956 & 0.000 & 0.000 & 0.000 & 0.406 & 0.000 & 0.000 & 0.000 & $\left\{Q_{1}\right\}$ \\
$Y_{1} \mid Y_{4}$ & 0.945 & 0.000 & 0.494 & 0.000 & 0.408 & 0.000 & 0.003 & 0.000 & $\left\{Q_{1}, Q_{4}\right\}$ \\
$Y_{1} \mid Y_{5}$ & 0.972 & 0.158 & 0.151 & 0.149 & 0.432 & 0.000 & 0.000 & 0.000 & $\left\{Q_{1}, Q_{2}, Q_{4}, Q_{5}\right\}$ \\
$Y_{1} \mid Y_{2}, Y_{3}$ & 0.862 & 0.759 & 0.000 & 0.000 & 0.376 & 0.004 & 0.000 & 0.000 & $\left\{Q_{1}, Q_{2}\right\}$ \\
$Y_{1} \mid Y_{2}, Y_{4}$ & 0.855 & 0.798 & 0.395 & 0.000 & 0.382 & 0.004 & 0.002 & 0.000 & $\left\{Q_{1}, Q_{2}, Q_{4}\right\}$ \\
$Y_{1} \mid Y_{2}, Y_{5}$ & 0.911 & 0.857 & 0.119 & 0.117 & 0.400 & 0.003 & 0.000 & 0.000 & $\left\{Q_{1}, Q_{2}, Q_{4}, Q_{5}\right\}$ \\
$Y_{1} \mid Y_{3}, Y_{4}$ & 0.867 & 0.000 & 0.766 & 0.000 & 0.382 & 0.000 & 0.003 & 0.000 & $\left\{Q_{1}, Q_{4}\right\}$ \\
$Y_{1} \mid Y_{3}, Y_{5}$ & 0.903 & 0.116 & 0.143 & 0.106 & 0.406 & 0.000 & 0.000 & 0.000 & $\left\{Q_{1}, Q_{2}, Q_{4}, Q_{5}\right\}$ \\
$Y_{1} \mid Y_{4}, Y_{5}$ & 0.908 & 0.191 & 0.452 & 0.178 & 0.412 & 0.000 & 0.003 & 0.000 & $\left\{Q_{1}, Q_{2}, Q_{4}, Q_{5}\right\}$ \\
$Y_{1} \mid Y_{2}, Y_{3}, Y_{4}$ & 0.731 & 0.651 & 0.638 & 0.000 & 0.360 & 0.004 & 0.003 & 0.000 & $\left\{Q_{1}, Q_{2}, Q_{4}\right\}$ \\
$Y_{1} \mid Y_{2}, Y_{3}, Y_{5}$ & 0.810 & 0.700 & 0.110 & 0.067 & 0.372 & 0.003 & 0.000 & 0.000 & $\left\{Q_{1}, Q_{2}, Q_{4}, Q_{5}\right\}$ \\
$Y_{1} \mid Y_{2}, Y_{4}, Y_{5}$ & 0.831 & 0.752 & 0.407 & 0.175 & 0.378 & 0.004 & 0.002 & 0.000 & $\left\{Q_{1}, Q_{2}, Q_{4}, Q_{5}\right\}$ \\
$Y_{1} \mid Y_{3}, Y_{4}, Y_{5}$ & 0.817 & 0.107 & 0.714 & 0.084 & 0.379 & 0.000 & 0.003 & 0.000 & $\left\{Q_{1}, Q_{2}, Q_{4}, Q_{5}\right\}$ \\
$Y_{1} \mid Y_{2}, Y_{3}, Y_{4}, Y_{5}$ & 0.734 & 0.650 & 0.636 & 0.000 & 0.354 & 0.003 & 0.003 & 0.000 & $\left\{Q_{1}, Q_{2}, Q_{4}\right\}$ \\
\hline
\end{tabular}

Table S 1: Frequencies of QTL detection for all possible conditional mapping analysis of $Y_{1}$. Results based on 1,000 simulated data sets described in the text. The expected architecture is the set of d-connected QTLs for the phenotype conditioning with respect to the network in Figure 1. 

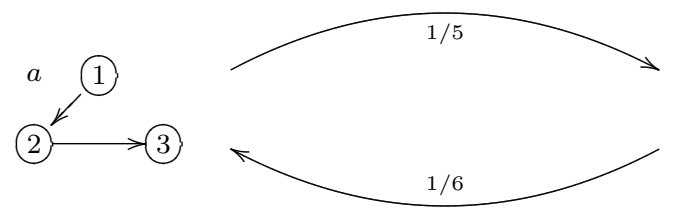

neighbourhood
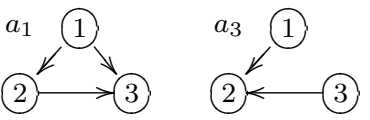

reverse

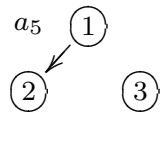

delete

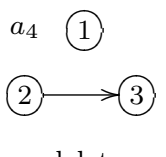

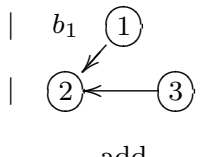

add

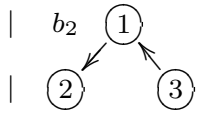

add

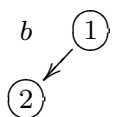

(3)

neighbourhood

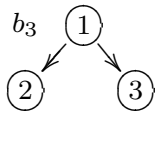

$b_{5}$

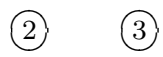

delete

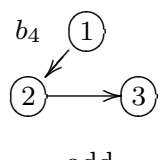

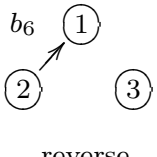

Figure S 1: Adapted from Husmeier (2003). Metropolis-Hastings proposal moves and ratio. This figure illustrates the three proposal modifications: adding, deleting or reversing one arrow in the network structure. When adding or reversing an arrow we need to check if the proposed modification leads to a cyclic network such (as in graph $a_{2}$ ). Cyclic structures are not valid and need to be discarded. The

Metropolis-Hastings proposal ratio $q\left(\mathcal{M}_{\text {old }} \mid \mathcal{M}_{\text {new }}\right) / q\left(\mathcal{M}_{\text {new }} \mid \mathcal{M}_{\text {old }}\right)$ is given by the ratio of the neighborhood sizes of the two networks involved in the proposal modification. The neighborhood of a DAG, $n e(\mathcal{M})$ is defined as the number of DAGs that can be obtained from the present DAG in a single proposal modification. As an example, let structure a be $\mathcal{M}_{\text {old }}$. Since structure $a_{2}$ is cyclic, ne $\left(\mathcal{M}_{\text {old }}\right)=5$ and the $q\left(\mathcal{M}_{\text {new }} \mid \mathcal{M}_{\text {old }}\right)=1 / 5$. Suppose we sampled structure $a_{5}$ so that $a_{5}=b=\mathcal{M}_{\text {new }}$. Then ne $\left(\mathcal{M}_{\text {new }}\right)=6$ and the $q\left(\mathcal{M}_{\text {old }} \mid \mathcal{M}_{\text {new }}\right)=1 / 6$. In this case the Metropolis-Hastings proposal ratio is $5 / 6$.

\begin{tabular}{|c|c|c|c|c|c|c|c|c|c|c|}
\hline \multirow{2}{*}{\multicolumn{2}{|c|}{ Phenotypes }} & \multicolumn{4}{|c|}{ strong signal } & \multicolumn{4}{|c|}{ weak signal } & \multirow{2}{*}{$\begin{array}{c}\text { Expected } \\
\text { architecture }\end{array}$} \\
\hline & & $Q_{1}$ & $Q_{2}$ & $Q_{4}$ & $Q_{5}$ & $Q_{1}$ & $Q_{2}$ & $Q_{4}$ & $Q_{5}$ & \\
\hline$Y_{2}$ & & 0.884 & 0.930 & 0.000 & 0.000 & 0.001 & 0.384 & 0.000 & 0.000 & $\left\{Q_{1}, Q_{2}\right\}$ \\
\hline & $Y_{1}$ & 0.000 & 0.999 & 0.000 & 0.000 & 0.000 & 0.424 & 0.000 & 0.000 & $\left\{Q_{2}\right\}$ \\
\hline$Y_{2}$ & $Y_{3}$ & 0.375 & 0.986 & 0.000 & 0.000 & 0.001 & 0.390 & 0.000 & 0.000 & $\left\{Q_{1}, Q_{2}\right\}$ \\
\hline$Y_{2}$ & $Y_{4}$ & 0.498 & 0.968 & 0.096 & 0.000 & 0.001 & 0.392 & 0.000 & 0.000 & $\left\{Q_{1}, Q_{2}, Q_{4}\right\}$ \\
\hline$Y_{2}$ & $Y_{5}$ & 0.500 & 0.850 & 0.192 & 0.232 & 0.001 & 0.360 & 0.000 & 0.000 & $\left\{Q_{1}, Q_{2}, Q_{4}, Q_{5}\right\}$ \\
\hline$Y_{2}$ & $Y_{1}, Y_{3}$ & 0.000 & 0.999 & 0.000 & 0.000 & 0.000 & 0.424 & 0.000 & 0.000 & $\left\{Q_{2}\right\}$ \\
\hline$Y_{2}$ & $Y_{1}, Y_{4}$ & 0.000 & 0.999 & 0.000 & 0.000 & 0.000 & 0.426 & 0.000 & 0.000 & $\left\{Q_{2}\right\}$ \\
\hline$Y_{2}$ & $Y_{1}, Y_{5}$ & 0.000 & 0.995 & 0.134 & 0.206 & 0.000 & 0.405 & 0.000 & 0.003 & $\left\{Q_{2}, Q_{4}, Q_{5}\right\}$ \\
\hline$Y_{2}$ & $Y_{3}, Y_{4}$ & 0.144 & 0.994 & 0.115 & 0.000 & 0.001 & 0.397 & 0.000 & 0.000 & $\left\{Q_{1}, Q_{2}, Q_{4}\right\}$ \\
\hline$Y_{2}$ & $Y_{3}, Y_{5}$ & 0.223 & 0.910 & 0.347 & 0.427 & 0.002 & 0.364 & 0.000 & 0.003 & $\left\{Q_{1}, Q_{2}, Q_{4}, Q_{5}\right\}$ \\
\hline$Y_{2}$ & $Y_{4}, Y_{5}$ & 0.253 & 0.826 & 0.180 & 0.686 & 0.001 & 0.357 & 0.000 & 0.003 & $\left\{Q_{1}, Q_{2}, Q_{4}, Q_{5}\right\}$ \\
\hline$Y_{2}$ & $Y_{1}, Y_{3}, Y_{4}$ & 0.000 & 0.999 & 0.000 & 0.000 & 0.000 & 0.426 & 0.000 & 0.000 & $\left\{Q_{2}\right\}$ \\
\hline$Y_{2}$ & $Y_{1}, Y_{3}, Y_{5}$ & 0.000 & 0.991 & 0.336 & 0.421 & 0.000 & 0.404 & 0.000 & 0.004 & $\left\{Q_{2}, Q_{4}, Q_{5}\right\}$ \\
\hline$Y_{2}$ & $Y_{1}, Y_{4}, Y_{5}$ & 0.000 & 0.988 & 0.035 & 0.698 & 0.000 & 0.405 & 0.000 & 0.004 & $\left\{Q_{2}, Q_{4}, Q_{5}\right\}$ \\
\hline$Y_{2}$ & $Y_{3}, Y_{4}, Y_{5}$ & 0.020 & 0.827 & 0.027 & 0.963 & 0.002 & 0.361 & 0.000 & 0.006 & $\left\{Q_{1}, Q_{2}, Q_{4}, Q_{5}\right\}$ \\
\hline$Y_{2}$ & $Y_{1}, Y_{3}, Y_{4}, Y_{5}$ & 0.000 & 0.969 & 0.000 & 0.937 & 0.000 & 0.402 & 0.000 & 0.007 & $\left\{Q_{2}, Q_{5}\right\}$ \\
\hline
\end{tabular}

Table S 2: Frequencies of QTL detection for all possible conditional mapping analysis of $Y_{2}$. Results based on 1,000 simulated data sets described in the text. The expected architecture is the set of d-connected QTLs for the phenotype conditioning with respect to the network in Figure 1. 


\begin{tabular}{|c|c|c|c|c|c|c|c|c|c|c|}
\hline \multirow{2}{*}{\multicolumn{2}{|c|}{ Phenotypes }} & \multicolumn{4}{|c|}{ strong signal } & \multicolumn{4}{|c|}{ weak signal } & \multirow{2}{*}{$\begin{array}{c}\text { Expected } \\
\text { architecture }\end{array}$} \\
\hline & & $Q_{1}$ & $Q_{2}$ & $Q_{4}$ & $Q_{5}$ & $Q_{1}$ & $Q_{2}$ & $Q_{4}$ & $Q_{5}$ & \\
\hline$Y_{3}$ & & 0.941 & 0.000 & 0.000 & 0.000 & 0.003 & 0.000 & 0.000 & 0.000 & $\left\{Q_{1}\right\}$ \\
\hline$Y_{3}$ & $Y_{1}$ & 0.000 & 0.000 & 0.000 & 0.000 & 0.000 & 0.000 & 0.000 & 0.000 & $\emptyset$ \\
\hline$Y_{3}$ & $Y_{2}$ & 0.525 & 0.503 & 0.000 & 0.000 & 0.003 & 0.000 & 0.000 & 0.000 & $\left\{Q_{1}, Q_{2}\right\}$ \\
\hline$Y_{3}$ & $Y_{4}$ & 0.478 & 0.000 & 0.688 & 0.000 & 0.005 & 0.000 & 0.003 & 0.000 & $\left\{Q_{1}, Q_{4}\right\}$ \\
\hline$Y_{3}$ & $Y_{5}$ & 0.546 & 0.212 & 0.228 & 0.258 & 0.003 & 0.000 & 0.000 & 0.001 & $\left\{Q_{1}, Q_{2}, Q_{4}, Q_{5}\right\}$ \\
\hline$Y_{3}$ & $Y_{1}, Y_{2}$ & 0.000 & 0.000 & 0.000 & 0.000 & 0.000 & 0.000 & 0.000 & 0.000 & $\emptyset$ \\
\hline$Y_{3}$ & $Y_{1}, Y_{4}$ & 0.000 & 0.000 & 0.916 & 0.000 & 0.000 & 0.000 & 0.001 & 0.000 & $\left\{Q_{4}\right\}$ \\
\hline$Y_{3}$ & $Y_{1}, Y_{5}$ & 0.000 & 0.199 & 0.214 & 0.218 & 0.000 & 0.000 & 0.000 & 0.001 & $\left\{Q_{2}, Q_{4}, Q_{5}\right\}$ \\
\hline$Y_{3}$ & $Y_{2}, Y_{4}$ & 0.318 & 0.315 & 0.747 & 0.000 & 0.003 & 0.000 & 0.003 & 0.000 & $\left.1, Q_{2}, Q_{4}\right\}$ \\
\hline$Y_{3}$ & $Y_{2}, Y_{5}$ & 0.290 & 0.275 & 0.379 & 0.421 & 0.003 & 0.000 & 0.000 & 0.001 & $\left\{Q_{1}, Q_{2}, Q_{4}, Q_{5}\right\}$ \\
\hline$Y_{3}$ & $Y_{4}, Y_{5}$ & 0.309 & 0.228 & 0.631 & 0.283 & 0.002 & 0.000 & 0.002 & 0.001 & $\left\{Q_{1}, Q_{2}, Q_{4}, Q_{5}\right\}$ \\
\hline$Y_{3}$ & $Y_{1}, Y_{2}, Y_{4}$ & 0.000 & 0.000 & 0.914 & 0.000 & 0.000 & 0.000 & 0.001 & 0.000 & $\{0\}$, \\
\hline$Y_{3}$ & $Y_{1}, Y_{2}, Y_{5}$ & 0.000 & 0.000 & 0.377 & 0.413 & 0.000 & 0.000 & 0.000 & 0.001 & $\left\{Q_{4}, Q_{5}\right\}$ \\
\hline$Y_{3}$ & $Y_{1}, Y_{4}, Y_{5}$ & 0.000 & 0.135 & 0.840 & 0.217 & 0.000 & 0.000 & 0.001 & 0.002 & $\left.Q_{2}, Q_{4}, Q_{5}\right\}$ \\
\hline$Y_{3}$ & $Y_{2}, Y_{4}, Y_{5}$ & 0.156 & 0.157 & 0.568 & 0.857 & 0.001 & 0.000 & 0.002 & 0.002 & $\left\{Q_{1}, Q_{2}, Q_{4}, Q_{5}\right\}$ \\
\hline$Y_{3}$ & $Y_{1}, Y_{2}, Y_{4}, Y_{5}$ & 0.000 & 0.000 & 0.764 & 0.762 & 0.000 & 0.000 & 0.001 & 0.002 & $\left\{Q_{4}, Q_{5}\right\}$ \\
\hline
\end{tabular}

Table S 3: Frequencies of QTL detection for all possible conditional mapping analysis of $Y_{3}$. Results based on 1,000 simulated data sets described in the text. The expected architecture is the set of d-connected QTLs for the phenotype conditioning with respect to the network in Figure 1.

\begin{tabular}{l|cccc|cccc|c}
\hline & \multicolumn{4}{|c|}{ strong signal } & \multicolumn{4}{c|}{ weak signal } & Expected \\
Phenotypes & $Q_{1}$ & $Q_{2}$ & $Q_{4}$ & $Q_{5}$ & $Q_{1}$ & $Q_{2}$ & $Q_{4}$ & $Q_{5}$ & architecture \\
\hline$Y_{4}$ & 0.603 & 0.000 & 0.690 & 0.000 & 0.003 & 0.000 & 0.370 & 0.000 & $\left\{Q_{1}, Q_{4}\right\}$ \\
$Y_{4} \mid Y_{1}$ & 0.000 & 0.000 & 0.960 & 0.000 & 0.000 & 0.000 & 0.392 & 0.000 & $\left\{Q_{4}\right\}$ \\
$Y_{4} \mid Y_{2}$ & 0.373 & 0.383 & 0.808 & 0.000 & 0.000 & 0.000 & 0.373 & 0.000 & $\left\{Q_{1}, Q_{2}, Q_{4}\right\}$ \\
$Y_{4} \mid Y_{3}$ & 0.372 & 0.000 & 0.979 & 0.000 & 0.002 & 0.000 & 0.401 & 0.000 & $\left\{Q_{1}, Q_{4}\right\}$ \\
$Y_{4} \mid Y_{5}$ & 0.383 & 0.330 & 0.607 & 0.385 & 0.002 & 0.000 & 0.348 & 0.005 & $\left\{Q_{1}, Q_{2}, Q_{4}, Q_{5}\right\}$ \\
$Y_{4} \mid Y_{1}, Y_{2}$ & 0.000 & 0.000 & 0.960 & 0.000 & 0.000 & 0.000 & 0.392 & 0.000 & $\left\{Q_{4}\right\}$ \\
$Y_{4} \mid Y_{1}, Y_{3}$ & 0.000 & 0.000 & 0.999 & 0.000 & 0.000 & 0.000 & 0.422 & 0.000 & $\left\{Q_{4}\right\}$ \\
$Y_{4} \mid Y_{1}, Y_{5}$ & 0.000 & 0.357 & 0.827 & 0.411 & 0.000 & 0.000 & 0.374 & 0.003 & $\left\{Q_{2}, Q_{4}, Q_{5}\right\}$ \\
$Y_{4} \mid Y_{2}, Y_{3}$ & 0.125 & 0.114 & 0.988 & 0.000 & 0.001 & 0.000 & 0.402 & 0.000 & $\left\{Q_{1}, Q_{2}, Q_{4}\right\}$ \\
$Y_{4} \mid Y_{2}, Y_{5}$ & 0.174 & 0.153 & 0.626 & 0.672 & 0.000 & 0.000 & 0.352 & 0.007 & $\left\{Q_{1}, Q_{2}, Q_{4}, Q_{5}\right\}$ \\
$Y_{4} \mid Y_{3}, Y_{5}$ & 0.205 & 0.357 & 0.905 & 0.450 & 0.001 & 0.000 & 0.376 & 0.001 & $\left\{Q_{1}, Q_{2}, Q_{4}, Q_{5}\right\}$ \\
$Y_{4} \mid Y_{1}, Y_{2}, Y_{3}$ & 0.000 & 0.000 & 0.999 & 0.000 & 0.000 & 0.000 & 0.420 & 0.000 & $\left\{Q_{4}\right\}$ \\
$Y_{4} \mid Y_{1}, Y_{2}, Y_{5}$ & 0.000 & 0.000 & 0.759 & 0.728 & 0.000 & 0.000 & 0.373 & 0.004 & $\left\{Q_{4}, Q_{5}\right\}$ \\
$Y_{4} \mid Y_{1}, Y_{3}, Y_{5}$ & 0.000 & 0.359 & 0.987 & 0.456 & 0.000 & 0.000 & 0.402 & 0.001 & $\left\{Q_{2}, Q_{4}, Q_{5}\right\}$ \\
$Y_{4} \mid Y_{2}, Y_{3}, Y_{5}$ & 0.013 & 0.017 & 0.836 & 0.973 & 0.000 & 0.000 & 0.377 & 0.003 & $\left\{Q_{1}, Q_{2}, Q_{4}, Q_{5}\right\}$ \\
$Y_{4} \mid Y_{1}, Y_{2}, Y_{3}, Y_{5}$ & 0.000 & 0.000 & 0.971 & 0.950 & 0.000 & 0.000 & 0.401 & 0.002 & $\left\{Q_{4}, Q_{5}\right\}$ \\
\hline
\end{tabular}

Table S 4: Frequencies of QTL detection for all possible conditional mapping analysis of $Y_{4}$. Results based on 1,000 simulated data sets described in the text. The expected architecture is the set of d-connected QTLs for the phenotype conditioning with respect to the network in Figure 1.

\begin{tabular}{|c|c|c|c|c|c|c|c|c|c|c|}
\hline \multirow{2}{*}{\multicolumn{2}{|c|}{ Phenotypes }} & \multicolumn{4}{|c|}{ strong signal } & \multicolumn{4}{|c|}{ weak signal } & \multirow{2}{*}{$\begin{array}{c}\text { Expected } \\
\text { architecture }\end{array}$} \\
\hline & & $Q_{1}$ & $Q_{2}$ & $Q_{4}$ & $Q_{5}$ & $Q_{1}$ & $Q_{2}$ & $Q_{4}$ & $Q_{5}$ & \\
\hline$Y_{5}$ & & 0.637 & 0.321 & 0.320 & 0.340 & 0.000 & 0.000 & 0.001 & 0.336 & $\left\{Q_{1}, Q_{2}, Q_{4}, Q_{5}\right\}$ \\
\hline$Y_{5}$ & $Y_{1}$ & 0.000 & 0.540 & 0.524 & 0.582 & 0.000 & 0.000 & 0.001 & 0.338 & $\left\{Q_{2}, Q_{4}, Q_{5}\right\}$ \\
\hline$Y_{5}$ & $Y_{2}$ & 0.296 & 0.272 & 0.546 & 0.566 & 0.000 & 0.000 & 0.001 & 0.350 & $\left\{Q_{1}, Q_{2}, Q_{4}, Q_{5}\right\}$ \\
\hline$Y_{5}$ & $Y_{3}$ & 0.242 & 0.584 & 0.598 & 0.652 & 0.000 & 0.000 & 0.001 & 0.362 & $\left\{Q_{1}, Q_{2}, Q_{4}, Q_{5}\right\}$ \\
\hline$Y_{5}$ & $Y_{4}$ & 0.280 & 0.669 & 0.221 & 0.744 & 0.000 & 0.001 & 0.000 & 0.377 & $\left\{Q_{1}, Q_{2}, Q_{4}, Q_{5}\right\}$ \\
\hline$Y_{5}$ & $Y_{1}, Y_{2}$ & 0.000 & 0.000 & 0.657 & 0.708 & 0.000 & 0.000 & 0.001 & 0.357 & $\left\{Q_{4}, Q_{5}\right\}$ \\
\hline$Y_{5}$ & $Y_{1}, Y_{3}$ & 0.000 & 0.701 & 0.708 & 0.809 & 0.000 & 0.000 & 0.001 & 0.367 & $\left\{Q_{2}, Q_{4}, Q_{5}\right\}$ \\
\hline$Y_{5}$ & $Y_{1}, Y_{4}$ & 0.000 & 0.793 & 0.130 & 0.893 & 0.000 & 0.001 & 0.000 & 0.369 & $\left.Q_{2}, Q_{4}, Q_{5}\right\}$ \\
\hline$Y_{5}$ & $Y_{2}, Y_{3}$ & 0.045 & 0.033 & 0.853 & 0.874 & 0.000 & 0.000 & 0.001 & 0.389 & $\left\{Q_{1}, Q_{2}, Q_{4}, Q_{5}\right\}$ \\
\hline$Y_{5}$ & $Y_{2}, Y_{4}$ & 0.118 & 0.093 & 0.283 & 0.968 & 0.000 & 0.000 & 0.000 & 0.395 & $\left\{Q_{1}, Q_{2}, Q_{4}, Q_{5}\right\}$ \\
\hline$Y_{5}$ & $Y_{3}, Y_{4}$ & 0.051 & 0.846 & 0.026 & 0.892 & 0.000 & 0.001 & 0.000 & 0.392 & $\left\{Q_{1}, Q_{2}, Q_{4}, Q_{5}\right\}$ \\
\hline$Y_{5}$ & $Y_{1}, Y_{2}, Y_{3}$ & 0.000 & 0.001 & 0.894 & 0.928 & 0.000 & 0.000 & 0.001 & 0.392 & $\left\{Q_{4}, Q_{5}\right\}$ \\
\hline$Y_{5}$ & $Y_{1}, Y_{2}, Y_{4}$ & 0.000 & 0.000 & 0.271 & 0.988 & 0.000 & 0.000 & 0.000 & 0.394 & $\left\{Q_{4}, Q_{5}\right\}$ \\
\hline$Y_{5}$ & $Y_{1}, Y_{3}, Y_{4}$ & 0.000 & 0.893 & 0.000 & 0.936 & 0.000 & 0.001 & 0.000 & 0.392 & $\left\{Q_{2}, Q_{5}\right\}$ \\
\hline$Y_{5}$ & $Y_{2}, Y_{3}, Y_{4}$ & 0.000 & 0.000 & 0.000 & 0.999 & 0.000 & 0.000 & 0.000 & 0.415 & $\left\{Q_{5}\right\}$ \\
\hline$Y_{5}$ & $Y_{1}, Y_{2}, Y_{3}, Y_{4}$ & 0.000 & 0.000 & 0.000 & 0.999 & 0.000 & 0.000 & 0.000 & 0.417 & $\left\{Q_{5}\right\}$ \\
\hline
\end{tabular}

Table S 5: Frequencies of QTL detection for all possible conditional mapping analysis of $Y_{5}$. Results based on 1,000 simulated data sets described in the text. The expected architecture is the set of d-connected QTLs for the phenotype conditioning with respect to the network in Figure 1 (main text). 


\begin{tabular}{|c|c|c|c|c|c|c|c|c|c|c|c|c|c|c|}
\hline & Apbb. & Clec2 & Trpv2 & D13E. & Pscd. & Frzb & Myo1f & Il10. & Unc5a & Aif1 & Riken & Stat4 & Il16 & Tnfs. \\
\hline Apbb. & 1 & 0.881 & 0.854 & 0.776 & 0.815 & 0.604 & 0.754 & 0.718 & 0.789 & 0.750 & 0.698 & 0.657 & 0.649 & 0.486 \\
\hline Clec2 & 0.860 & 1 & 0.852 & 0.739 & 0.704 & 0.642 & 0.736 & 0.776 & 0.708 & 0.651 & 0.735 & 0.604 & 0.614 & 0.442 \\
\hline Trpv2 & 0.827 & 0.825 & 1 & 0.782 & 0.709 & 0.597 & 0.783 & 0.750 & 0.807 & 0.693 & 0.687 & 0.587 & 0.587 & 0.536 \\
\hline D13E. & 0.732 & 0.691 & 0.739 & 1 & 0.764 & 0.751 & 0.734 & 0.663 & 0.741 & 0.619 & 0.678 & 0.650 & 0.728 & 0.571 \\
\hline Pscd. & 0.777 & 0.644 & 0.645 & 0.708 & 1 & 0.594 & 0.781 & 0.595 & 0.664 & 0.699 & 0.591 & 0.619 & 0.648 & 0.554 \\
\hline Frzb & 0.519 & 0.570 & 0.510 & 0.693 & 0.484 & 1 & 0.601 & 0.631 & 0.500 & 0.371 & 0.700 & 0.462 & 0.545 & 0.442 \\
\hline Myolf & 0.705 & 0.686 & 0.740 & 0.676 & 0.727 & 0.506 & 1 & 0.673 & 0.764 & 0.747 & 0.702 & 0.566 & 0.545 & 0.585 \\
\hline Il10. & 0.666 & 0.737 & 0.704 & 0.597 & 0.506 & 0.552 & 0.608 & 1 & 0.689 & 0.587 & 0.694 & 0.481 & 0.670 & 0.402 \\
\hline Unc5a & 0.749 & 0.656 & 0.770 & 0.688 & 0.588 & 0.389 & 0.715 & 0.630 & 1 & 0.732 & 0.681 & 0.580 & 0.567 & 0.567 \\
\hline Aif1 & 0.698 & 0.582 & 0.629 & 0.532 & 0.620 & 0.212 & 0.688 & 0.501 & 0.675 & 1 & 0.528 & 0.621 & 0.586 & 0.636 \\
\hline Riken & 0.637 & 0.685 & 0.624 & 0.607 & 0.488 & 0.627 & 0.636 & 0.633 & 0.614 & 0.417 & 1 & 0.490 & 0.525 & 0.471 \\
\hline Stat4 & 0.594 & 0.534 & 0.512 & 0.581 & 0.535 & 0.346 & 0.479 & 0.386 & 0.501 & 0.542 & 0.387 & 1 & 0.559 & 0.624 \\
\hline Il16 & 0.581 & 0.543 & 0.507 & 0.672 & 0.566 & 0.442 & 0.449 & 0.607 & 0.481 & 0.494 & 0.423 & 0.474 & 1 & 0.509 \\
\hline Tnfs. & 0.377 & 0.330 & 0.438 & 0.473 & 0.437 & 0.301 & 0.489 & 0.276 & 0.473 & 0.547 & 0.346 & 0.546 & 0.401 & 1 \\
\hline
\end{tabular}

Table S 6: Correlations between phenotypes. The upper triangle gives the pairwise correlations between phenotypes. The lower triangle shows the pairwise correlations between the residuals of the phenotypes after adjustment for the common QTL to which all phenotypes map in the unconditional analysis.

\begin{tabular}{|c|c|c|c|c|c|c|c|c|c|}
\hline pheno & pheno & $\rightarrow$ & $\leftarrow$ & $\not t, t$ & pheno & pheno & $\rightarrow$ & $\leftarrow$ & $\not \rightarrow, 4$ \\
\hline Apbb. & Clec2 2 & 1.000 & 0.000 & 0.000 & Pscd. & Frzb & 0.000 & 0.302 & 0.698 \\
\hline Apbb. & Trpv2 & 0.581 & 0.000 & 0.419 & Pscd. & Myo1f & 0.969 & 0.031 & 0.000 \\
\hline Apbb. & D13E. & 0.186 & 0.005 & 0.809 & Pscd. & Illo. & 0.186 & 0.083 & 0.731 \\
\hline Apbb. & Pscd. & 1.000 & 0.000 & 0.000 & Pscd. & Unc5a & 0.058 & 0.071 & 0.871 \\
\hline Apbb. & Frzb & 0.000 & 0.998 & 0.002 & Pscd. & Aif1 & 0.17 & 0.162 & 0.668 \\
\hline Apbb. & Myo1f & 0.259 & 0.000 & 0.741 & Pscd. & Riken & 0.075 & 0.048 & 0.877 \\
\hline Apbb. & Illo. & 0.748 & 0.000 & 0.252 & Pscd. & Stat4 & 0.067 & 0.057 & 0.876 \\
\hline Apbb. & Unc5a & 0.998 & 0.000 & 0.002 & Pscd. & Il16 & 0.005 & 0.488 & 0.507 \\
\hline Apbb. & Aif1 & 1.000 & 0.000 & 0.000 & Pscd. & Tnfs. & 0.324 & 0.409 & 0.267 \\
\hline Apbb. & Riken & 0.250 & 0.000 & 0.750 & Frzb & Myo1f & 0.284 & 0.000 & 0.716 \\
\hline Apbb. & Stat 4 & 0.908 & 0.077 & 0.015 & Frzb & Illo. & 0.548 & 0.000 & 0.452 \\
\hline Apbb. & Il16 & 0.006 & 0.983 & 0.011 & Frzb & Unc5a & 0.074 & 0.000 & 0.926 \\
\hline Apbb. & Tnfs. & 0.144 & 0.038 & 0.818 & Frzb & Aif1 & 0.932 & 0.000 & 0.068 \\
\hline Clec2 & Trpv2 & 0.874 & 0.123 & 0.003 & Frzb & Riken & 1.000 & 0.000 & 0.000 \\
\hline Clec2 & D13E. & 0.201 & 0.013 & 0.786 & Frzb & Stat4 & 0.066 & 0.000 & 0.934 \\
\hline Clec2 & Pscd. & 0.087 & 0.094 & 0.819 & Frzb & Il16 & 0.005 & 0.984 & 0.011 \\
\hline Clec2 & Frzb & 0.000 & 0.718 & 0.282 & Frzb & Tnfs. & 0.199 & 0.066 & 0.735 \\
\hline Clec2 & Myo1f & 0.122 & 0.022 & 0.856 & Myo1f & Il10. & 0.089 & 0.570 & 0.341 \\
\hline Clec2 & Il10. & 0.838 & 0.093 & 0.069 & Myolf & Unc5a & 0.086 & 0.540 & 0.374 \\
\hline Clec2 & Unc5a & 0.083 & 0.113 & 0.804 & Myo1f & Aif1 & 0.006 & 0.152 & 0.842 \\
\hline Clec2 & Aif1 & 0.089 & 0.035 & 0.876 & Myo1f & Riken & 0.117 & 0.568 & 0.315 \\
\hline Clec2 & Riken & 0.810 & 0.170 & 0.020 & Myo1f & Stat4 & 0.028 & 0.166 & 0.806 \\
\hline Clec2 & Stat4 & 0.065 & 0.044 & 0.891 & Myolf & Il16 & 0.001 & 0.511 & 0.488 \\
\hline Clec2 & Il1 16 & 0.000 & 0.051 & 0.949 & Myo1f & Tnfs. & 0.054 & 0.442 & 0.504 \\
\hline Clec2 & Tnfs. & 0.048 & 0.054 & 0.898 & Il10. & Unc5a & 0.034 & 0.552 & 0.414 \\
\hline Trpv2 & D13E. & 0.954 & 0.033 & 0.013 & Illo. & Aif1 & 0.442 & 0.127 & 0.431 \\
\hline Trpv2 & Pscd. & 0.076 & 0.114 & 0.810 & Il10. & Riken & 0.075 & 0.475 & 0.450 \\
\hline Trpv2 & Frzb & 0.000 & 0.117 & 0.883 & Il10. & Stat 4 & 0.048 & 0.132 & 0.820 \\
\hline Trpv2 & Myolf & 0.968 & 0.015 & 0.017 & Il10. & Il16 & 0.002 & 0.998 & 0.000 \\
\hline Trpv2 & Illo. & 0.966 & 0.011 & 0.023 & Il10. & Tnfs. & 0.011 & 0.294 & 0.695 \\
\hline Trpv2 & Unc5a & 0.059 & 0.916 & 0.025 & Unc5a & Aif1 & 0.214 & 0.310 & 0.476 \\
\hline Trpv2 & Aif1 & 0.116 & 0.054 & 0.830 & Unc5a & Riken & 0.789 & 0.173 & 0.038 \\
\hline Trpv2 & Riken & 0.109 & 0.08 & 0.811 & Unc5a & Stat 4 & 0.044 & 0.058 & 0.898 \\
\hline Trpv2 & Stat4 & 0.065 & 0.089 & 0.846 & Unc5a & Il16 & 0.001 & 0.059 & 0.94 \\
\hline Trpv2 & Il16 & 0.002 & 0.059 & 0.939 & Unc5a & Tnfs. & 0.005 & 0.931 & 0.064 \\
\hline Trpv2 & Tnfs. & 0.026 & 0.222 & 0.752 & Aif1 & Riken & 0.101 & 0.143 & 0.756 \\
\hline D13E. & Pscd. & 0.081 & 0.893 & 0.026 & Aif1 & Stat4 & 0.042 & 0.074 & 0.884 \\
\hline D13E. & Frzb & 0.000 & 1.000 & 0.000 & Aif1 & Il16 & 0.004 & 0.123 & 0.873 \\
\hline D13E. & Myo1f & 0.275 & 0.212 & 0.513 & Aif1 & Tnfs. & 0.074 & 0.926 & 0.000 \\
\hline D13E. & Illo. & 0.132 & 0.750 & 0.118 & Riken & Stat4 & 0.037 & 0.109 & 0.854 \\
\hline D13E. & Unc5a & 0.005 & 0.367 & 0.628 & Riken & Il16 & 0.001 & 0.097 & 0.902 \\
\hline D13E. & Aif1 & 0.096 & 0.211 & 0.693 & Riken & Tnfs. & 0.040 & 0.099 & 0.861 \\
\hline D13E. & Riken & 0.077 & 0.182 & 0.741 & Stat4 & Il16 & 0.031 & 0.210 & 0.759 \\
\hline D13E. & Stat4 & 0.010 & 0.959 & 0.031 & Stat4 & Tnfs. & 0.239 & 0.761 & 0.000 \\
\hline D13E. & Il16 & 0.007 & 0.993 & 0.000 & Il16 & Tnfs. & 0.430 & 0.192 & 0.378 \\
\hline D13E. & Tnfs. & 0.009 & 0.144 & 0.847 & & & & & \\
\hline
\end{tabular}

Table S 7: Posterior probabilities for causal relationships computed via Bayesian model averaging. 
3. Convergence diagnostics for the real data example. We assess the convergence of the Markov chain with Geweke (1992) and Raftery and Lewis (1992, 1995) diagnostic measures, and by trace and autocorrelation plots. Inspection of the trace plots suggest good mixing of the Markov chain and the autocorrelation plot corroborates this finding (Figure S2).

Geweke's convergence diagnostic is based on a test for equality of the means of the first and last part of a Markov chain (we used the first 10\% and the last $50 \%$ parts of the Markov chain after dropping the burnin). When the MCMC samples are drawn from the stationary distribution of the chain, the two means are equal, and Geweke's statistic is asymptotically $N(0,1)$. Our test statistic (a standard Z-score) was 0.7685 , suggesting that convergence was achieved.

The Raftery and Lewis diagnostic is a run length control diagnostic based on the accuracy of estimation of the quantile $q$. It estimates: (1) the size of burnin (4 for our chain); (2) the required MCMC sample size (the estimate was 5,107 and our chain had size 9,000); and (3) the dependency factor that measures the extent to which autocorrelation inflates the required sample size (the estimated dependency factor was 1.36. Values above 5 suggest problems due to high autocorrelation). These results again suggest that convergence was achieved.
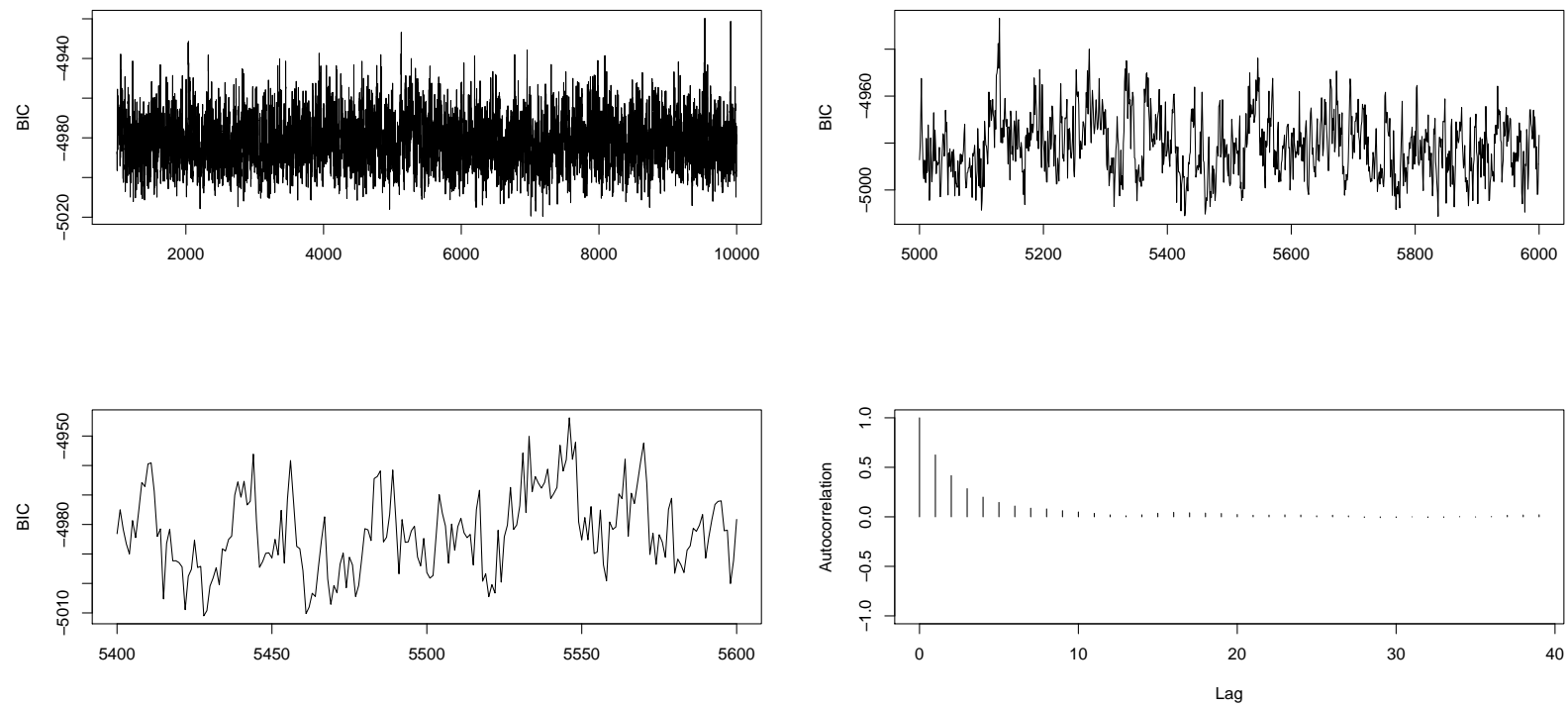

Figure S 2: Trace and autocorrelation plots for the Markov chain. The y-axis on the top and left bottom panels shows the BIC for each sampled network. The top left plot displays the Markov chain for the 9,000 samples structures. The top right and bottom left plots show windows of size 1,000 and 200, respectively.

The bottom right plot shows the autocorrelation plot.

4. Comparison with Winrow et al. In this section we compare the QTLnet algorithm with the strategy proposed by Winrow et al. (2009). We evaluated the performance of Winrow's strategy in the same simulated data sets of our original simulation study. In order to make the comparison of our strategy (re-mapping and M-H applied to phenotype nodes) and Winrow's (single mapping and M-H applied to both phenotype and QTL nodes) more direct, we scored the networks using the BIC approximation to the marginal likelihood, instead of the Bayesian scoring metric proposed by 
Bøttcher (2005) and adopted in Winrow et al. Furthermore, Winrow et al. enforce some constraints to the M-H proposal mechanism, including the following: (i) each phenotype node is allowed to be affected by at most two parent phenotypes (although the number of QTL parents is kept free); and (ii) phenotypes are not allowed to be parents of QTLs; and (iii) QTLs are not allowed to be parents of QTLs; and (iv) edges between QTL and phenotypes that are not found to be controlled by those respective QTL in the unconditional mapping analysis are dropped. In our simulations we only enforced conditions (ii) and (iii).

Table S8 shows that QTLnet outperforms Winrow et al. in terms of genetic architecture inference and phenotype network recovery in the strong signal setting. In the weak signal setting both approaches are comparable.

\begin{tabular}{|c|c|c|c|c|c|c|c|c|c|c|c|c|}
\hline \multirow[b]{3}{*}{ Pairs } & \multicolumn{6}{|c|}{ Strong signal } & \multicolumn{6}{|c|}{ Weak signal } \\
\hline & \multicolumn{3}{|c|}{ QTLnet } & \multicolumn{3}{|c|}{ Winrow et al. } & \multicolumn{3}{|c|}{ QTLnet } & \multicolumn{3}{|c|}{ Winrow et al. } \\
\hline & $\rightarrow$ & $\leftarrow$ & $\nrightarrow, \leftarrow$ & $\rightarrow$ & $\leftarrow$ & $\not, t$ & $\rightarrow$ & $\leftarrow$ & $\not, \leftarrow$ & $\rightarrow$ & $\leftarrow$ & $\nrightarrow, \leftarrow$ \\
\hline$\left(Y_{1}, Y_{2}\right)$ & 0.996 & 0.002 & 0.002 & 0.989 & 0.011 & 0.000 & 0.594 & 0.177 & 0.229 & 0.581 & 0.183 & 0.236 \\
\hline$\left(Y_{1}, Y_{3}\right)$ & 0.990 & 0.002 & 0.008 & 0.885 & 0.011 & 0.104 & 0.471 & 0.263 & 0.266 & 0.451 & 0.269 & 0.280 \\
\hline$\left(Y_{1}, Y_{4}\right)$ & 0.990 & 0.001 & 0.009 & 0.966 & 0.017 & 0.017 & 0.541 & 0.196 & 0.263 & 0.496 & 0.220 & 0.284 \\
\hline$\left(Y_{1}, Y_{5}\right)$ & 0.054 & 0.002 & 0.944 & 0.037 & 0.018 & 0.945 & 0.028 & 0.005 & 0.967 & 0.019 & 0.005 & 0.976 \\
\hline$\left(Y_{2}, Y_{3}\right)$ & 0.016 & 0.022 & 0.962 & 0.050 & 0.020 & 0.930 & 0.018 & 0.017 & 0.965 & 0.007 & 0.011 & 0.982 \\
\hline$\left(Y_{2}, Y_{4}\right)$ & 0.037 & 0.012 & 0.951 & 0.056 & 0.019 & 0.925 & 0.018 & 0.015 & 0.967 & 0.011 & 0.008 & 0.981 \\
\hline$\left(Y_{2}, Y_{5}\right)$ & 0.997 & 0.003 & 0.000 & 0.976 & 0.022 & 0.002 & 0.712 & 0.075 & 0.213 & 0.699 & 0.078 & 0.223 \\
\hline$\left(Y_{3}, Y_{4}\right)$ & 0.967 & 0.031 & 0.002 & 0.768 & 0.210 & 0.022 & 0.482 & 0.253 & 0.265 & 0.475 & 0.247 & 0.278 \\
\hline$\left(Y_{3}, Y_{5}\right)$ & 0.997 & 0.003 & 0.000 & 0.955 & 0.042 & 0.003 & 0.653 & 0.116 & 0.231 & 0.631 & 0.118 & 0.251 \\
\hline$\left(Y_{4}, Y_{5}\right)$ & 0.996 & 0.004 & 0.000 & 0.959 & 0.038 & 0.003 & 0.670 & 0.115 & 0.215 & 0.641 & 0.140 & 0.219 \\
\hline$\left(Q_{1}, Y_{1}\right)$ & 0.997 & 0.000 & 0.003 & 0.993 & 0.000 & 0.007 & 0.402 & 0.000 & 0.598 & 0.430 & 0.000 & 0.570 \\
\hline$\left(Q_{1}, Y_{2}\right)$ & 0.002 & 0.000 & 0.998 & 0.008 & 0.000 & 0.992 & 0.002 & 0.000 & 0.998 & 0.000 & 0.000 & 1.000 \\
\hline$\left(Q_{1}, Y_{3}\right)$ & 0.000 & 0.000 & 1.000 & 0.012 & 0.000 & 0.988 & 0.000 & 0.000 & 1.000 & 0.000 & 0.000 & 1.000 \\
\hline$\left(Q_{1}, Y_{4}\right)$ & 0.009 & 0.000 & 0.991 & 0.009 & 0.000 & 0.991 & 0.001 & 0.000 & 0.999 & 0.000 & 0.000 & 1.000 \\
\hline$\left(Q_{1}, Y_{5}\right)$ & 0.001 & 0.000 & 0.999 & 0.015 & 0.000 & 0.985 & 0.001 & 0.000 & 0.999 & 0.001 & 0.000 & 0.999 \\
\hline$\left(Q_{2}, Y_{1}\right)$ & 0.002 & 0.000 & 0.998 & 0.017 & 0.000 & 0.983 & 0.000 & 0.000 & 1.000 & 0.000 & 0.000 & 1.000 \\
\hline$\left(Q_{2}, Y_{2}\right)$ & 0.998 & 0.000 & 0.002 & 0.935 & 0.000 & 0.065 & 0.394 & 0.000 & 0.606 & 0.384 & 0.000 & 0.616 \\
\hline$\left(Q_{2}, Y_{3}\right)$ & 0.000 & 0.000 & 1.000 & 0.002 & 0.000 & 0.998 & 0.000 & 0.000 & 1.000 & 0.000 & 0.000 & 1.000 \\
\hline$\left(Q_{2}, Y_{4}\right)$ & 0.001 & 0.000 & 0.999 & 0.006 & 0.000 & 0.994 & 0.000 & 0.000 & 1.000 & 0.000 & 0.000 & 1.000 \\
\hline$\left(Q_{2}, Y_{5}\right)$ & 0.001 & 0.000 & 0.999 & 0.021 & 0.000 & 0.979 & 0.000 & 0.000 & 1.000 & 0.000 & 0.000 & 1.000 \\
\hline$\left(Q_{4}, Y_{1}\right)$ & 0.001 & 0.000 & 0.999 & 0.016 & 0.000 & 0.984 & 0.000 & 0.000 & 1.000 & 0.001 & 0.000 & 0.999 \\
\hline$\left(Q_{4}, Y_{2}\right)$ & 0.000 & 0.000 & 1.000 & 0.003 & 0.000 & 0.997 & 0.000 & 0.000 & 1.000 & 0.002 & 0.000 & 0.998 \\
\hline$\left(Q_{4}, Y_{3}\right)$ & 0.000 & 0.000 & 1.000 & 0.041 & 0.000 & 0.959 & 0.000 & 0.000 & 1.000 & 0.000 & 0.000 & 1.000 \\
\hline$\left(Q_{4}, Y_{4}\right)$ & 0.990 & 0.000 & 0.010 & 0.724 & 0.000 & 0.276 & 0.387 & 0.000 & 0.613 & 0.370 & 0.000 & 0.630 \\
\hline$\left(Q_{4}, Y_{5}\right)$ & 0.003 & 0.000 & 0.997 & 0.024 & 0.000 & 0.976 & 0.000 & 0.000 & 1.000 & 0.000 & 0.000 & 1.000 \\
\hline$\left(Q_{5}, Y_{1}\right)$ & 0.000 & 0.000 & 1.000 & 0.005 & 0.000 & 0.995 & 0.000 & 0.000 & 1.000 & 0.000 & 0.000 & 1.000 \\
\hline$\left(Q_{5}, Y_{2}\right)$ & 0.002 & 0.000 & 0.998 & 0.006 & 0.000 & 0.994 & 0.000 & 0.000 & 1.000 & 0.000 & 0.000 & 1.000 \\
\hline$\left(Q_{5}, Y_{3}\right)$ & 0.000 & 0.000 & 1.000 & 0.005 & 0.000 & 0.995 & 0.000 & 0.000 & 1.000 & 0.000 & 0.000 & 1.000 \\
\hline$\left(Q_{5}, Y_{4}\right)$ & 0.003 & 0.000 & 0.997 & 0.010 & 0.000 & 0.990 & 0.000 & 0.000 & 1.000 & 0.000 & 0.000 & 1.000 \\
\hline$\left(Q_{5}, Y_{5}\right)$ & 0.998 & 0.000 & 0.002 & 0.340 & 0.000 & 0.660 & 0.389 & 0.000 & 0.611 & 0.336 & 0.000 & 0.664 \\
\hline
\end{tabular}

Table S 8: Comparison of QTLnet versus the approach proposed by Winrow et al. (2009).

Although Winrow's strategy also avoids the detection of indirect QTLs, it is prone to miss direct QTLs when the causal effects among phenotypes are strong. To illustrate this point, consider Table 1 on Section 6. When the causal signal is strong, $Y_{5}$ mapped to $Q_{1}$ more frequently $(63.7 \%)$ than to $Q_{5}(34 \%)$ since $Y_{5}$ has stronger correlation with $Q_{1}$ than to $Q_{5}$. This suggests that in strong signal settings, we can potentially miss the direct QTLs of downstream traits. However, when we perform the mapping analysis of $Y_{5}$ conditional on its phenotype parents, $Q_{5}$ is detected in $99.9 \%$ of the simulated data sets.

Table S8 shows that although Winrow's strategy often failed to detect $Q_{4}$ and $Q_{5}$ as direct QTL 
for $Y_{4}$ and $Y_{5}$, it still performed well in terms of inference of the causal direction between $Y_{4}$ and $Y_{5}$. This example illustrates how phenotypes are sometimes enough to break likelihood equivalence (note that because $Y_{1}$ affects $Y_{4}$ but not $Y_{5}$, and $Y_{2}$ affects $Y_{5}$ but not $Y_{4}$, we still can learn the causal direction between $Y_{4}$ and $Y_{5}$ when we miss $Q_{4}$ and $Q_{5}$ ).

However, missing direct QTLs may have a serious impact depending on the structure of the phenotype network. For instance, suppose the true network is

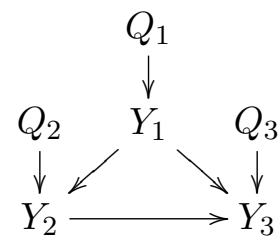

and assume that the sample size, the residual variances and the regression coefficients are, respectively, $n=100, \sigma^{2}=3$ and $\beta=2$ (see the last paragraph in this section for details on the regression models and computation of LOD scores). Unconditional mapping analysis of $Y_{1}$ detects $Q_{1}$ $\left(\operatorname{LOD}\left(Y_{1}, Q_{1}\right)=15.05\right)$. Unconditional mapping analysis of $Y_{2}$ detects $Q_{1}\left(\operatorname{LOD}\left(Y_{2}, Q_{1}\right)=11.09\right)$ but not $Q_{2}\left(L O D\left(Y_{2}, Q_{2}\right)=2.29\right)$. Finally, unconditional mapping analysis of $Y_{3}$ detects $Q_{1}$ $\left(\operatorname{LOD}\left(Y_{3}, Q_{1}\right)=12.55\right)$ but not $Q_{3}\left(\operatorname{LOD}\left(Y_{3}, Q_{3}\right)=0.27\right)$ or $Q_{2}\left(\operatorname{LOD}\left(Y_{3}, Q_{2}\right)=1.09\right)$. Application of the M-H to $Q_{1}, Y_{1}, Y_{2}$ and $Y_{3}$ allows the inference of causal directions $Q_{1} \rightarrow Y_{1}$, $Y_{1} \rightarrow Y_{2}$ and $Y_{1} \rightarrow Y_{3}$. However, the causal direction between $Y_{2}$ and $Y_{3}$ cannot be learned from the data since models
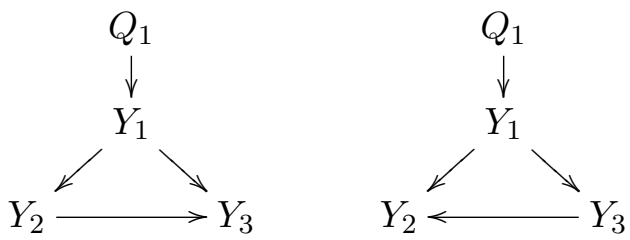

are likelihood equivalent.

QTLnet is not susceptible to this problem since conditional mapping analysis of $Y_{2}$ given $Y_{1}$ detects $Q_{2}\left(L O D\left(Y_{2}, Q_{2} \mid Y_{1}\right)=15.05\right)$ and the conditional mapping analysis of $Y_{3}$ given $\left\{Y_{1}, Y_{2}\right\}$ detects $Q_{3}\left(L O D\left(Y_{3}, Q_{3} \mid Y_{1}, Y_{2}\right)=15.05\right)$.

Computation of the LOD scores: let $Y$ and $\{Q, \mathbf{C}\}$ represent the independent and dependent variables in a regression model with standard Gaussian errors. The LOD score is given by $L O D(Y, Q, \mathbf{C})=(n / 2) \log _{10}\left\{1 /\left(1-R^{2}(Y, Q, \mathbf{C})\right)\right\}$ where $R^{2}(Y, Q, \mathbf{C})=1-\operatorname{Var}(Y \mid Q, \mathbf{C}) / \operatorname{Var}(Y)$ corresponds to the multiple correlation coefficient of $Y$ and $\{Q, \mathbf{C}\}$. The conditional LOD score is defined as $\operatorname{LOD}(Y, Q \mid \mathbf{C})=\operatorname{LOD}(Y, Q, \mathbf{C})-\operatorname{LOD}(Y, \mathbf{C})$. Assuming only additive and dominance effects for the QTLs, the DAG given in (4.1) factorizes in three regression models $Y_{1}=$ $\beta_{a 1} A_{1}+\beta_{d 1} D_{1}+\epsilon_{1}, Y_{2}=\beta_{21} Y_{1}+\beta_{a 2} A_{2}+\beta_{d 2} D_{2}+\epsilon_{2}$ and $Y_{3}=\beta_{31} Y_{1}+\beta_{32} Y_{2}+\beta_{a 3} A_{3}+\beta_{d 3} D_{3}+\epsilon_{3}$, where the $A$ s and $D$ s represent dummy variables coded according to Cockerham's genetic model. (In a $F_{2}$ experimental cross population the genotype distribution of any segregating locus $Q_{k}=\{b b, B b, B B\}$ is $(0.25,0.5,0.25)$. Cockerham's genetic model re-codes $Q_{k}$ into additive, $A_{k}=\#\{\mathrm{~b}$ alleles $\}-1$, and dominance, $D_{k}=\left(1+A_{k}\right)\left(1-A_{k}\right)-1 / 2$, dummy variables.) Assuming that the residual variances of the three models are equal to $\sigma^{2}$ and all regression coefficients are equal to $\beta$ we have that: $\operatorname{Var}\left(Y_{1}\right)=\sigma^{2}+3 \beta^{2} / 4 ; \operatorname{Var}\left(Y_{2}\right)=\left(\sigma^{2}+3 \beta^{2} / 4\right)\left(1+\beta^{2}\right) ; \operatorname{Var}\left(Y_{3}\right)=\left(\sigma^{2}+3 \beta^{2} / 4\right)\left(1+\beta^{2}+\right.$ $\left.\beta^{2}(1+\beta)^{2}\right) ; \operatorname{Var}\left(Y_{1} \mid Y_{2}\right)=\sigma^{2}+3 \beta^{2} / 4 ; \operatorname{Var}\left(Y_{3} \mid Y_{1}, Y_{2}\right)=\sigma^{2}+3 \beta^{2} / 4 ; \operatorname{Var}\left(Y_{1} \mid Q_{1}\right)=\sigma^{2}$, 
$\operatorname{Var}\left(Y_{2} \mid Q_{1}\right)=\sigma^{2}+\beta^{2}\left(\sigma^{2}+3 / 4\right) ; \operatorname{Var}\left(Y_{2} \mid Q_{2}\right)=\sigma^{2}+\beta^{2}\left(\sigma^{2}+3 \beta^{2} / 4\right) ; \operatorname{Var}\left(Y_{3} \mid Q_{1}\right)=$ $\sigma^{2} \beta^{2}(1+\beta)^{2}+\left(\sigma^{2}+3 \beta^{2} / 4\right)\left(1+\beta^{2}\right) ; \operatorname{Var}\left(Y_{3} \mid Q_{2}\right)=\beta^{2}(1+\beta)^{2}\left(\sigma^{2}+3 \beta^{2} / 4\right)+\sigma^{2}\left(1+\beta^{2}\right)+3 \beta^{2} / 4 ;$ $\operatorname{Var}\left(Y_{3} \mid Q_{3}\right)=\beta^{2}(1+\beta)^{2}\left(\sigma^{2}+3 \beta^{2} / 4\right)+\sigma^{2}\left(1+\beta^{2}\right)+3 \beta^{4} / 4 ; \operatorname{Var}\left(Y_{2} \mid Y_{1}, Q_{2}\right)=\sigma^{2}$; and $\operatorname{Var}\left(Y_{3} \mid Y_{1}, Y_{2}, Q_{3}\right)=\sigma^{2}$.

5. Proof of Result 1. Result 1: The conditional joint distribution of the phenotype traits organized according to the set of structural equations

$$
y_{t i}=\mu_{t i}^{\star}+\sum_{v \in p a\left(y_{t}\right)} \beta_{t v} y_{v i}+\epsilon_{t i}, \quad \epsilon_{t i} \sim N\left(0, \sigma_{t}^{2}\right),
$$

with $i=1, \ldots, n, t=1, \ldots, T$ and $\epsilon_{t i}$ independent error terms has distribution

$$
\mathbf{y}_{i} \mid \boldsymbol{\mu}_{i}^{\star}, \boldsymbol{\beta}, \boldsymbol{\sigma}^{2} \sim N_{T}\left(\boldsymbol{\Omega}^{-1} \gamma_{i}, \boldsymbol{\Omega}^{-1}\right),
$$

where $\boldsymbol{\mu}_{i}^{\star}=\left(\mu_{1 i}^{\star}, \ldots, \mu_{T i}^{\star}\right)^{\prime}, \boldsymbol{\beta}=\left\{\beta_{t v}: v \in p a\left(y_{t}\right), t=1, \ldots, T\right\}, \boldsymbol{\sigma}^{2}=\left(\sigma_{1}^{2}, \ldots, \sigma_{T}^{2}\right)^{\prime}$, $\boldsymbol{\Omega}$ is the concentration matrix with entries given by

$$
\omega_{t v}= \begin{cases}\frac{1}{\sigma_{t}^{2}}+\sum_{s} \frac{\beta_{s t}^{2}}{\sigma_{s}^{2}} \mathbb{1}_{\{t \rightarrow s\}}, & \text { for } t=v \\ -\frac{\beta_{v t}}{\sigma_{v}^{2}} \mathbb{1}_{\{t \rightarrow v\}}-\frac{\beta_{t v}}{\sigma_{t}^{2}} \mathbb{1}_{\{v \rightarrow t\}}+\sum_{s} \frac{\beta_{s v} \beta_{s t}}{\sigma_{v}^{2}} \mathbb{1}_{\{v \rightarrow s, t \rightarrow s\}}, & \text { for } t \neq v\end{cases}
$$

$\gamma_{i}$ is a vector with entries

$$
\frac{\mu_{t i}^{\star}}{\sigma_{t}^{2}}-\sum_{s \neq t} \frac{\beta_{s t} \mu_{s i}^{\star}}{\sigma_{s}^{2}} \mathbb{1}_{\{t \rightarrow s\}}
$$

and $\mathbb{1}_{\{t \rightarrow s\}}$ is the indicator function that trait $t$ affects trait $s$.

We start by proving some algebraic equalities needed in the proof of Result 1 .

Result 5: Let $\mathbb{1}\{j \rightarrow k\}$ represent the indicator function that $y_{j} \in p a\left(y_{k}\right)$ or, conversely, that $y_{k} \in \operatorname{ch}\left(y_{j}\right)$. Then

(a)

$$
\sum_{t=1}^{T} \lambda_{t} \mu_{t}^{\star}\left(y_{t}-\sum_{k: y_{k} \in p a\left(y_{t}\right)} \beta_{t k} y_{k}\right)=\sum_{t=1}^{T}\left(\lambda_{t} \mu_{t}^{\star}-\sum_{s \neq t} \lambda_{s} \beta_{s t} \mu_{s}^{\star} \mathbb{1}_{\{t \rightarrow s\}}\right) y_{t} .
$$

$$
\sum_{t=1}^{T} \lambda_{t}\left(y_{t}^{2}+\sum_{k: y_{k} \in p a\left(y_{t}\right)} \beta_{t k}^{2} y_{k}^{2}\right)=\sum_{t=1}^{T}\left(\lambda_{t}+\sum_{s \neq t} \lambda_{s} \beta_{s t}^{2} \mathbb{1}_{\{t \rightarrow s\}}\right) y_{t}^{2} .
$$

$$
\begin{aligned}
& \sum_{t=1}^{T} \lambda_{t}\left(-\sum_{k: y_{k} \in p a\left(y_{t}\right)} \beta_{t k} y_{t} y_{k}+\sum_{(k, j): y_{k}, y_{j} \in p a\left(y_{t}\right)} \beta_{t k} \beta_{t j} y_{k} y_{j}\right)= \\
& =\sum_{v<t}\left(-\lambda_{v} \beta_{v t} \mathbb{1}_{\{t \rightarrow v\}}-\lambda_{t} \beta_{t v} \mathbb{1}_{\{v \rightarrow t\}}+\sum_{s \neq v, s \neq t} \lambda_{s} \beta_{s v} \beta_{s t} \mathbb{1}_{\{v \rightarrow s, t \rightarrow s\}}\right) y_{v} y_{t} .
\end{aligned}
$$


All three items can be proved by induction. We only present the proof for item (b); the other proofs are similar.

Proof. The result holds for $T=3$

$$
\begin{aligned}
& \sum_{t=1}^{3} \lambda_{t}\left(y_{t}^{2}+\sum_{k: y_{k} \in p a\left(y_{t}\right)} \beta_{t k}^{2} y_{k}^{2}\right)=\sum_{t=1}^{3} \lambda_{t}\left(y_{t}^{2}+\sum_{k \neq t} \beta_{t k}^{2} \mathbb{1}_{\{k \rightarrow t\}} y_{k}^{2}\right)= \\
& =\lambda_{1}\left(y_{1}^{2}+\beta_{12}^{2} y_{2}^{2} \mathbb{1}_{\{2 \rightarrow 1\}}+\beta_{13}^{2} y_{3}^{2} \mathbb{1}_{\{3 \rightarrow 1\}}\right)+\lambda_{2}\left(y_{2}^{2}+\beta_{21}^{2} y_{1}^{2} \mathbb{1}_{\{1 \rightarrow 2\}}+\beta_{23}^{2} y_{3}^{2} \mathbb{1}_{\{3 \rightarrow 2\}}\right) \\
& +\lambda_{3}\left(y_{3}^{2}+\beta_{31}^{2} y_{1}^{2} \mathbb{1}_{\{1 \rightarrow 3\}}+\beta_{32}^{2} y_{2}^{2} \mathbb{1}_{\{2 \rightarrow 3\}}\right)=\left(\lambda_{1}+\lambda_{2} \beta_{21}^{2} \mathbb{1}_{\{1 \rightarrow 2\}}+\lambda_{3} \beta_{31}^{2} \mathbb{1}_{\{1 \rightarrow 3\}}\right) y_{1}^{2} \\
& +\left(\lambda_{2}+\lambda_{1} \beta_{12}^{2} \mathbb{1}_{\{2 \rightarrow 1\}}+\lambda_{3} \beta_{32}^{2} \mathbb{1}_{\{2 \rightarrow 3\}}\right) y_{2}^{2}+\left(\lambda_{3}+\lambda_{1} \beta_{13}^{2} \mathbb{1}_{\{3 \rightarrow 1\}}+\lambda_{2} \beta_{32}^{2} \mathbb{1}_{\{3 \rightarrow 2\}}\right) y_{3}^{2} \\
& =\sum_{t=1}^{3}\left(\lambda_{t}+\sum_{s \neq t} \lambda_{s} \beta_{s t}^{2} \mathbb{1}_{\{t \rightarrow s\}}\right) y_{t}^{2} .
\end{aligned}
$$

Relabeling 1,2 and 3 by $T-1, T$ and $T+1$ it follows that

$$
\sum_{t=T-1}^{T+1} \lambda_{t}\left(y_{t}^{2}+\sum_{k: y_{k} \in p a\left(y_{t}\right)} \beta_{t k}^{2} y_{k}^{2}\right)=\sum_{t=T-1}^{T+1}\left(\lambda_{t}+\sum_{s \neq t} \lambda_{s} \beta_{s t}^{2} \mathbb{1}_{\{t \rightarrow s\}}\right) y_{t}^{2} .
$$

The result also holds taking the summation from $T-1$ to $T$ (it is easy to show the result for $T=2$ ). Now assume the result holds for $T$

$$
\sum_{t=1}^{T} \lambda_{t}\left(y_{t}^{2}+\sum_{k: y_{k} \in p a\left(y_{t}\right)} \beta_{t k}^{2} y_{k}^{2}\right)=\sum_{t=1}^{T}\left(\lambda_{t}+\sum_{s \neq t} \lambda_{s} \beta_{s t}^{2} \mathbb{1}_{\{t \rightarrow s\}}\right) y_{t}^{2} .
$$

Next we show that the result holds for $T+1$. Summing $\lambda_{T+1}\left(y_{T+1}^{2}+\sum_{k: y_{k} \in p a\left(y_{T+1}\right)} \beta_{T+1, k}^{2} y_{k}^{2}\right)$ on both sides of $(5.2)$

$$
\begin{aligned}
& \sum_{t=1}^{T} \lambda_{t}\left(y_{t}^{2}+\sum_{k: y_{k} \in p a\left(y_{t}\right)} \beta_{t k}^{2} y_{k}^{2}\right)+\lambda_{T+1}\left(y_{T+1}^{2}+\sum_{k: y_{k} \in p a\left(y_{T+1}\right)} \beta_{T+1, k}^{2} y_{k}^{2}\right) \\
& =\sum_{t=1}^{T}\left(\lambda_{t}+\sum_{s \neq t} \lambda_{s} \beta_{s t}^{2} \mathbb{1}_{\{t \rightarrow s\}}\right) y_{t}^{2}+\lambda_{T+1}\left(y_{T+1}^{2}+\sum_{k: y_{k} \in p a\left(y_{T+1}\right)} \beta_{T+1, k}^{2} y_{k}^{2}\right) \\
& =\sum_{t=1}^{T-2}\left(\lambda_{t}+\sum_{s \neq t} \lambda_{s} \beta_{s t}^{2} \mathbb{1}_{\{t \rightarrow s\}}\right) y_{t}^{2}+\sum_{t=T-1}^{T}\left(\lambda_{t}+\sum_{s \neq t} \lambda_{s} \beta_{s t}^{2} \mathbb{1}_{\{t \rightarrow s\}}\right) y_{t}^{2} \\
& +\lambda_{T+1}\left(y_{T+1}^{2}+\sum_{k: y_{k} \in p a\left(y_{T+1}\right)} \beta_{T+1, k}^{2} y_{k}^{2}\right)
\end{aligned}
$$

and from (5.1) 


$$
\begin{aligned}
=\sum_{t=1}^{T-2}\left(\lambda_{t}+\sum_{s \neq t} \lambda_{s} \beta_{s t}^{2} \mathbb{1}_{\{t \rightarrow s\}}\right) y_{t}^{2}+ & \sum_{t=T-1}^{T} \lambda_{t}\left(y_{t}^{2}+\sum_{k: y_{k} \in p a\left(y_{t}\right)} \beta_{t k}^{2} y_{k}^{2}\right) \\
& +\lambda_{T+1}\left(y_{T+1}^{2}+\sum_{k: y_{k} \in p a\left(y_{T+1}\right)} \beta_{T+1, k}^{2} y_{k}^{2}\right) \\
= & \sum_{t=1}^{T-2}\left(\lambda_{t}+\sum_{s \neq t} \lambda_{s} \beta_{s t}^{2} \mathbb{1}_{\{t \rightarrow s\}}\right) y_{t}^{2}+\sum_{t=T-1}^{T+1} \lambda_{t}\left(y_{t}^{2}+\sum_{k: y_{k} \in p a\left(y_{t}\right)} \beta_{t k}^{2} y_{k}^{2}\right)
\end{aligned}
$$

and from (5.1) again

$$
\begin{aligned}
& =\sum_{t=1}^{T-2}\left(\lambda_{t}+\sum_{s \neq t} \lambda_{s} \beta_{s t}^{2} \mathbb{1}_{\{t \rightarrow s\}}\right) y_{t}^{2}+\sum_{t=T-1}^{T+1}\left(\lambda_{t}+\sum_{s \neq t} \lambda_{s} \beta_{s t}^{2} \mathbb{1}_{\{t \rightarrow s\}}\right) y_{t}^{2} \\
& =\sum_{t=1}^{T+1}\left(\lambda_{t}+\sum_{s \neq t} \lambda_{s} \beta_{s t}^{2} \mathbb{1}_{\{t \rightarrow s\}}\right) y_{t}^{2} .
\end{aligned}
$$

Proof. (Result 1)

A set of discrete (q) and continuous (y) variables follow a conditional Gaussian distribution (Lauritzen 1996) if the joint distribution of the vectors of discrete and continuous variables have a density $f$ such that

$$
\log f(\mathbf{q}, \mathbf{y})=g(\mathbf{q})+\mathbf{h}^{\prime}(\mathbf{q}) \mathbf{y}-\mathbf{y}^{\prime} \mathbf{K}(\mathbf{q}) \mathbf{y} / 2,
$$

where $g(\mathbf{q})$ is a scalar, $\mathbf{h}(\mathbf{q})$ is a vector and $\mathbf{K}(\mathbf{q})$ is a positive definite matrix. The above statement is equivalent to

$$
P(\mathbf{q})>0 \quad \text { and } \quad(\mathbf{y} \mid \mathbf{q}) \sim N\left(\mathbf{K}(\mathbf{q})^{-1} \mathbf{h}(\mathbf{q}), \mathbf{K}(\mathbf{q})^{-1}\right) .
$$

In the following we will rewrite $\log p(\mathbf{y}, \mathbf{q} \mid \mathbf{m})$ (note we dropped the index $i$ from the notation) in the format of (5.3) and we will obtain the explicit forms of $g(\mathbf{q}), \mathbf{h}(\mathbf{q})$ and $\mathbf{K}(\mathbf{q})$. Let $\boldsymbol{\epsilon}=\left(\epsilon_{1}, \ldots, \epsilon_{T}\right)^{\prime}$ be the vector of independent normal error terms and $\mathbf{y}=\left(y_{1}, \ldots, y_{T}\right)^{\prime}$ the traits. Denote the Jacobian element for the transformation from $\boldsymbol{\epsilon} \rightarrow \mathbf{y}$ by $J_{\boldsymbol{\epsilon} \rightarrow \mathbf{y}}$. Then the joint density of the phenotype traits conditional on the respective genetic architectures is given by

$p(\mathbf{y} \mid \mathbf{q})=\prod_{t=1}^{T} p\left(y_{t} \mid \mathbf{q}_{t}, p a\left(y_{t}\right)\right)=\frac{\left|J_{\boldsymbol{\epsilon} \rightarrow \mathbf{y}}\right|}{(2 \pi)^{T / 2} \prod_{t=1}^{T} \sigma_{t}} \exp \left\{-\sum_{t=1}^{T} \frac{1}{2 \sigma_{t}^{2}}\left(y_{t}-\mu_{t}^{\star}-\sum_{y_{k} \in p a\left(y_{t}\right)} \beta_{t k} y_{k}\right)^{2}\right\}$,

where

$$
\left(y_{t}-\mu_{t}^{\star}-\sum_{y_{k} \in p a\left(y_{t}\right)} \beta_{t k} y_{k}\right)^{2}=\left(\mu_{t}^{\star 2}-2 \mu_{t}^{\star} y_{t}+2 \mu_{t}^{\star} \sum_{y_{k} \in p a\left(y_{t}\right)} \beta_{t k} y_{k}\right)+
$$




$$
+\left(y_{t}^{2}+\sum_{y_{k} \in p a\left(y_{t}\right)} \beta_{t k}^{2} y_{k}^{2}-2 \sum_{y_{k} \in p a\left(y_{t}\right)} \beta_{t k} y_{t} y_{k}+2 \sum_{k<j} \beta_{t k} \beta_{t j} y_{k} y_{j}\right)
$$

Let $\lambda_{t}=\prod_{j \neq t} \sigma_{j}^{2}$. Then

$$
\log p(\mathbf{y}, \mathbf{q} \mid \mathbf{m})=\log p(\mathbf{q} \mid \mathbf{m})+\log \left\{\frac{\left|J_{\boldsymbol{\epsilon} \rightarrow \mathbf{y}}\right|}{(2 \pi)^{T / 2} \prod_{t=1}^{T} \sigma_{t}}\right\}+(I)+(I I)
$$

where

$$
\begin{aligned}
(I) & =-\frac{1}{2 \prod_{t=1}^{T} \sigma_{t}^{2}} \sum_{t=1}^{T} \lambda_{t}\left(\mu_{t}^{\star 2}-2 \mu_{t}^{\star} y_{t}+2 \mu_{t}^{\star} \sum_{y_{k} \in p a\left(y_{t}\right)} \beta_{t k} y_{k}\right) \\
& \left.=-\frac{1}{2 \prod_{t=1}^{T} \sigma_{t}^{2}}\left[\sum_{t=1}^{T} \lambda_{t} \mu_{t}^{\star 2}-2 \sum_{t=1}^{T} \lambda_{t} \mu_{t}^{\star}\left(y_{t}-\sum_{y_{k} \in p a\left(y_{t}\right)} \beta_{t k} y_{k}\right)\right]\right] \\
& =-\frac{1}{2 \prod_{t=1}^{T} \sigma_{t}^{2}}\left[\sum_{t=1}^{T} \lambda_{t} \mu_{t}^{\star 2}-2 \sum_{t=1}^{T} y_{t}\left(\lambda_{t} \mu_{t}^{\star}-\sum_{s \neq t} \lambda_{s} \beta_{s t} \mu_{s}^{\star} \mathbb{1}_{\{t \rightarrow s\}}\right)\right] \\
& =-\frac{1}{2} \sum_{t=1}^{T} \frac{\mu_{t}^{\star 2}}{\sigma_{t}^{2}}+\sum_{t=1}^{T}\left(\frac{\mu_{t}^{\star}}{\sigma_{t}^{2}}-\sum_{s \neq t} \frac{\beta_{s t} \mu_{s}^{\star}}{\sigma_{s}^{2}} \mathbb{1}_{\{t \rightarrow s\}} y_{t}\right. \\
& =-\frac{1}{2} \sum_{t=1}^{T} \frac{\mu_{t}^{\star 2}}{\sigma_{t}^{2}}+\gamma^{\prime} \mathbf{y},
\end{aligned}
$$

where the third equality follows from Result 5 (a) and

$$
\begin{aligned}
& \gamma^{\prime}=\left(\frac{\mu_{1}^{\star}}{\sigma_{1}^{2}}-\sum_{s \neq 1} \frac{\beta_{s 1} \mu_{s}^{\star}}{\sigma_{s}^{2}} \mathbb{1}_{\{1 \rightarrow s\}}, \ldots, \frac{\mu_{T}^{\star}}{\sigma_{T}^{2}}-\sum_{s \neq T} \frac{\beta_{s T} \mu_{s}^{\star}}{\sigma_{s}^{2}} \mathbb{1}_{\{T \rightarrow s\}}\right) \\
& (I I)=-\frac{1}{2 \prod_{t=1}^{T} \sigma_{t}^{2}} \sum_{t=1}^{T} \lambda_{t}\left(y_{t}^{2}+\sum_{y_{k} \in p a\left(y_{t}\right)} \beta_{t k}^{2} y_{k}^{2}-2 \sum_{y_{k} \in p a\left(y_{t}\right)} \beta_{t k} y_{t} y_{k}+2 \sum_{k<j} \beta_{t k} \beta_{t j} y_{k} y_{j}\right) \\
& =-\frac{1}{2 \prod_{t=1}^{T} \sigma_{t}^{2}}\left[\sum_{t=1}^{T} \lambda_{t}\left(y_{t}^{2}+\sum_{y_{k} \in p a\left(y_{t}\right)} \beta_{t k}^{2} y_{k}^{2}\right)+\right. \\
& \left.+2 \sum_{t=1}^{T} \lambda_{t}\left(-\sum_{y_{k} \in p a\left(y_{t}\right)} \beta_{t k} y_{t} y_{k}+\sum_{k<j} \beta_{t k} \beta_{t j} y_{k} y_{j}\right)\right] \\
& =-\frac{1}{2 \prod_{t=1}^{T} \sigma_{t}^{2}}\left[\sum_{t=1}^{T}\left(\lambda_{t}+\sum_{s \neq t} \lambda_{s} \beta_{s t}^{2} \mathbb{1}_{\{t \rightarrow s\}}\right) y_{t}^{2}+\right.
\end{aligned}
$$




$$
\begin{aligned}
& \left.+2 \sum_{v<t}\left(-\lambda_{v} \beta_{v t} \mathbb{1}_{\{t \rightarrow v\}}-\lambda_{t} \beta_{t v} \mathbb{1}_{\{v \rightarrow t\}}+\sum_{s \neq v, s \neq t} \lambda_{s} \beta_{s v} \beta_{s t} \mathbb{1}_{\{v \rightarrow s, t \rightarrow s\}}\right) y_{v} y_{t}\right] \\
= & -\frac{1}{2}\left[\sum_{t=1}^{T}\left(\frac{1}{\sigma_{t}^{2}}+\sum_{s \neq t} \frac{\beta_{s t}^{2}}{\sigma_{s}^{2}} \mathbb{1}_{\{t \rightarrow s\}}\right) y_{t}^{2}+\right. \\
& \left.+2 \sum_{v<t}\left(-\frac{\beta_{v t}}{\sigma_{v}^{2}} \mathbb{1}_{\{t \rightarrow v\}}-\frac{\beta_{t v}}{\sigma_{t}^{2}} \mathbb{1}_{\{v \rightarrow t\}}+\sum_{s} \frac{\beta_{s v} \beta_{s t}}{\sigma_{v}^{2}} \mathbb{1}_{\{v \rightarrow s, t \rightarrow s\}}\right) y_{v} y_{t}\right] \\
= & -\frac{1}{2} \mathbf{y}^{\prime} \mathbf{\Omega} \mathbf{y},
\end{aligned}
$$

where the third equality follows from Result 5 (b) and (c) and $\boldsymbol{\Omega}$ is a concentration matrix with entries

$$
\omega_{t v}= \begin{cases}\frac{1}{\sigma_{t}^{2}}+\sum_{s} \frac{\beta_{s t}^{2}}{\sigma_{s}^{2}} \mathbb{1}_{\{t \rightarrow s\}}, & \text { for } t=v \\ -\frac{\beta_{v t}}{\sigma_{v}^{2}} \mathbb{1}_{\{t \rightarrow v\}}-\frac{\beta_{t v}}{\sigma_{t}^{2}} \mathbb{1}_{\{v \rightarrow t\}}+\sum_{s} \frac{\beta_{s v} \beta_{s t}}{\sigma_{v}^{2}} \mathbb{1}_{\{v \rightarrow s, t \rightarrow s\}}, & \text { for } t \neq v\end{cases}
$$

Therefore,

$$
\log p(\mathbf{q}, \mathbf{y} \mid \mathbf{m})=g(\mathbf{q})+\mathbf{h}^{\prime}(\mathbf{q}) \mathbf{y}-\mathbf{y}^{\prime} \mathbf{K}(\mathbf{q}) \mathbf{y} / 2
$$

with $\mathbf{K}(\mathbf{q})=\boldsymbol{\Omega}, \mathbf{h}(\mathbf{q})=\boldsymbol{\gamma}$ and $g(\mathbf{q})=\log p(\mathbf{q} \mid \mathbf{m})-\frac{1}{2}\left(T \log 2 \pi-\log \operatorname{det}(\boldsymbol{\Omega})+\sum_{t=1}^{T} \mu_{t}^{\star 2} / \sigma_{t}^{2}\right)$. Note that since $\boldsymbol{\Omega}$ is not a function of $\mathbf{q}$ our model is in the homogeneous conditional Gaussian parametric family (Lauritzen 1996). Finally, it follows from equation (5.4) that

$$
\mathbf{y} \mid \mathbf{q} \sim N_{T}\left(\mathbf{\Omega}^{-1} \gamma, \mathbf{\Omega}^{-1}\right)
$$

6. Proof of Result 2. Result 2: Consider a class of Markov equivalent DAGs $\mathcal{G}$. Let $Y_{1}$ and $Y_{2}$ be any two adjacent nodes in the graphs in $\mathcal{G}$. Assume that for each such pair there exists at least one variable, $Q$, directly affecting $Y_{1}$ but not $Y_{2}$. Let $\mathcal{G}_{E}$ represent the class of extended graphs. Then the graphs in $\mathcal{G}_{E}$ are not Markov equivalent.

Proof. Consider first subgraphs composed by $Y_{1}, Y_{2}$ and $Q$. Since $Q$ is a cause of $Y_{1}$ but not of $Y_{2}$, the three variables can only be represented by two causal subgraphs: $Q \rightarrow Y_{1} \leftarrow Y_{2}$ and $Q \rightarrow Y_{1} \rightarrow Y_{2}$. Since both subgraphs have the same skeleton, $Q-Y_{1}-Y_{2}$, but different sets of v-structures $\left(Q \rightarrow Y_{1} \leftarrow Y_{2}\right.$ for the first and no v-structure for the second) it follows from Theorem 1 that they are not Markov equivalent. Since by assumption this is true for any pair of adjacent nodes, $Y_{1}$ and $Y_{2}$, in any graph in $\mathcal{G}$, it follows that all graphs in $\mathcal{G}_{E}$ will have different sets of $\mathrm{v}$-structures, and the result follows. 
7. Proof of Result 3. Result 3: For the HCGR parametric family, two DAGs are distribution equivalent if and only if they are Markov equivalent.

Distribution equivalence implies Markov equivalence, but the converse is not generally true. For the linear regression with Gaussian errors parametric family, Markov equivalence does imply distribution equivalence (Heckerman and Geiger 1996). The proof for the HCGR model is a slightly modification of the proof for the Gaussian regression model. We therefore present the proof for latter case first. It is based on the following two results.

Theorem 2: Two DAGs are Markov equivalent if and only if there exists a set of covered arrow reversals that transform one structure into the other (Chickering, 1995).

An arrow reversal is a transformation from one DAG to the other where a single arrow between two nodes is reversed. An arrow between two nodes is said to be covered if the two nodes have the same parents when the arrow is removed. For example, the arrow from $Y_{1}$ to $Y_{2}$ is covered in graphs (a) and (b) but not in (c).

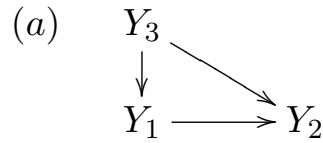
(b)

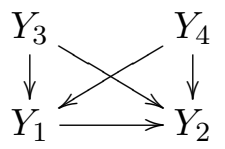

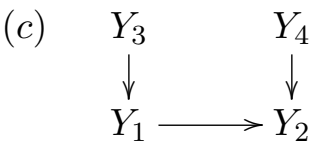

Theorem 3: Consider the Gaussian linear regression model with local likelihoods

$$
y_{t} \mid \mathbf{y}_{p a\left(y_{t}\right)} \sim N\left(\mu_{t}+\sum_{k \in p a\left(y_{t}\right)} \beta_{t k}\left(y_{k}-\mu_{k}\right), \sigma_{t}^{2}\right) .
$$

Given that node $j \in p a\left(y_{t}\right)$ and no other directed path exists from $j$ to $t$,

$$
\begin{aligned}
& E\left(y_{t} \mid \mathbf{y}_{K}\right)=\mu_{t}+\sum_{k \in K}\left(\beta_{t k}+\beta_{j k} \beta_{t j}\right)\left(y_{k}-\mu_{k}\right), \text { and } \\
& \operatorname{Var}\left(y_{t} \mid \mathbf{y}_{K}\right)=\sigma_{t}^{2}+\beta_{t j}^{2} \sigma_{j}^{2},
\end{aligned}
$$

where $K=\left(p a\left(y_{t}\right) \cup p a\left(y_{j}\right)\right) \backslash\left\{y_{j}\right\}$. If neither $\sigma_{j}^{2}$ or $\sigma_{t}^{2}$ are zero, then

$$
\begin{aligned}
& E\left(y_{j} \mid y_{t}, \mathbf{y}_{K}\right)=\mu_{j}+\sum_{k \in K}\left(\beta_{j k}-\left(\beta_{t k}+\beta_{j k} \beta_{t j}\right) \beta_{j t}^{*}\right)\left(y_{k}-\mu_{k}\right)+\beta_{j t}^{*}\left(y_{t}-\mu_{t}\right), \\
& \operatorname{Var}\left(y_{j} \mid y_{t}, \mathbf{y}_{K}\right)=\frac{\sigma_{j}^{2} \sigma_{t}^{2}}{\sigma_{t}^{2}+\beta_{t j}^{2} \sigma_{j}^{2}}, \quad \beta_{j t}^{*}=\frac{\beta_{t j} \sigma_{j}^{2}}{\sigma_{t}^{2}+\beta_{t j}^{2} \sigma_{j}^{2}} .
\end{aligned}
$$

with asterisk denoting the coefficients revised during the arrow reversal (Shachter and Kenley 1989, page 533).

The above Theorem shows that for the Gaussian linear regression parametric family a single covered arrow reversal is equivalent to a re-parametrization of the model. Consider for example graph (a). The joint distribution factors as $f\left(y_{3}\right) f\left(y_{1} \mid y_{3}\right) f\left(y_{2} \mid y_{1}, y_{3}\right)$ where $\left(y_{3}\right) \sim N\left(\mu_{3}, \sigma_{3}^{2}\right)$, $\left(y_{1} \mid y_{3}\right) \sim N\left(\mu_{1}+\beta_{13}\left(y_{3}-\mu_{3}\right), \sigma_{1}^{2}\right)$ and $\left(y_{2} \mid y_{1}, y_{3}\right) \sim N\left(\mu_{2}+\beta_{21}\left(y_{1}-\mu_{1}\right)+\beta_{23}\left(y_{3}-\mu_{3}\right), \sigma_{2}^{2}\right)$. 
Now let's consider the arrow reversal $Y_{1} \leftarrow Y_{2}$. From Theorem 3 we have $K=y_{3}$ and the components of re-parameterized model $f\left(y_{3}\right) f\left(y_{2} \mid y_{3}\right) f\left(y_{1} \mid y_{2}, y_{3}\right)$ normally distributed with expectations and variances

$$
\begin{aligned}
& E\left(y_{3}\right)=\mu_{3}, \quad \operatorname{Var}\left(y_{3}\right)=\sigma_{3}^{2}, \\
& E\left(y_{2} \mid y_{3}\right)=\mu_{2}+\left(\beta_{23}+\beta_{13} \beta_{21}\right)\left(y_{3}-\mu_{3}\right), \quad \operatorname{Var}\left(y_{2} \mid y_{3}\right)=\sigma_{2}^{2}+\beta_{21}^{2} \sigma_{1}^{2}, \\
& E\left(y_{1} \mid y_{2}, y_{3}\right)=\mu_{1}+\left(\beta_{13}-\left(\beta_{23}+\beta_{13} \beta_{21}\right) \frac{\beta_{21} \sigma_{1}^{2}}{\sigma_{2}^{2}+\beta_{21}^{2} \sigma_{1}^{2}}\right)\left(y_{3}-\mu_{3}\right)+\frac{\beta_{21} \sigma_{1}^{2}}{\sigma_{2}^{2}+\beta_{21}^{2} \sigma_{1}^{2}}\left(y_{2}-\mu_{2}\right), \\
& \operatorname{Var}\left(y_{1} \mid y_{2}, y_{3}\right)=\frac{\sigma_{1}^{2} \sigma_{2}^{2}}{\sigma_{2}^{2}+\beta_{21}^{2} \sigma_{1}^{2}} .
\end{aligned}
$$

We now present the proof, given by Heckerman and Geiger (1995), for the linear regression model with Gaussian errors.

Proof. We want to show that for the Gaussian linear regression parametric family two models are distribution equivalent if and only if they are Markov equivalent. Distribution equivalence implies Markov equivalence since if two models are distribution equivalent than one is reparametrization of the other, and the set of conditional independence/dependence relations entailed by them must be the same.

To show the other direction, that Markov equivalence implies distributional equivalence, note that by Theorem 2, if two structures are Markov equivalent, then there exists a set of covered arrow reversals that transform one structure into the other. Theorem 3, by its turn, implies that two structures differing by a set of covered arrow reversals are distribution equivalent.

We now present the proof for the HCGR model.

Proof. (Result 3) Since we only allow arrow reversals between phenotypes (but not between QTLs and phenotypes), and the QTLs enter the HCGR model through the mean, the result follows by replacing $\mu_{t}$ by $\mu_{t}^{*}=\mu_{t}+\mathbf{X}_{t} \boldsymbol{\theta}_{t}+\sum_{k \in p a\left(y_{t}\right)} \beta_{t k}\left(\mu_{k}+\mathbf{X}_{k} \boldsymbol{\theta}_{k}\right)$ and $\mu_{k}$ by $\mu_{k}^{\star}=\mu_{k}+\mathbf{X}_{k} \boldsymbol{\theta}_{k}$ in Theorem 3. (Note we are dropping the $i$ subscript from the notation in $\mathbf{X}_{t i}$ and $\mathbf{X}_{k i}$ ).

8. Proof of Result 4. Result 4: For the HCGR parametric family, two DAGs are distribution equivalent if and only if they have the same skeletons and same sets of v-structures.

Proof. Follows directly from Theorem 1 (main text) and Result 3.

\section{REFERENCES}

[1] Banerjee S, Yandell BS, Yi N (2008) Bayesian QTL mapping for multiple traits. Genetics 179: 2275 -2289.

[2] Bøтtсher SG (2005) Learning conditional Gaussian networks. Technical report R-2005-22. Aalborg University.

[3] Chickering D (1995) Search operators for learning equivalent classes of Bayesian network structures. Techinical Report R231, Cognitive Systems Laboratory, University of California, Los Angeles.

[4] GeweKe J (1992) Evaluating the accuracy of sampling-based approaches to calculating posterior moments. In Bayesian Statistics 4 (ed JM Bernado, JO Berger, AP Dawid and AFM Smith). Clarendon Press, Oxford, UK. 
[5] Heckerman D And Geiger D (1996) Likelihoods and parameter priors for Bayesian networks. Technical Report MSR-TR-95-94, Microsoft Research.

[6] Husmeier D (2003) Sensitivity and specificity of inferring genetic regulatory interactions from microarray experiments with dynamic Bayesian networks. Bioinformatics 19: 2271-2282.

[7] Kass RE, Raftery A (1995) Bayes factors. Journal of the American Statistical Association 90: 773-795.

[8] Raftery, AE AND Lewis, SM (1992). One long run with diagnostics: Implementation strategies for Markov chain Monte Carlo. Statistical Science 7, 493-497.

[9] Raftery, AE And Lewis, SM (1995). The number of iterations, convergence diagnostics and generic Metropolis algorithms. In Practical Markov Chain Monte Carlo (W.R. Gilks, D.J. Spiegelhalter and S. Richardson, eds.). London, U.K.: Chapman and Hall.

[10] Raftery AE, Newton MA, Satagopan JM, Krivitsky PN (2007) Estimating the integrated likelihood via posterior simulation using the harmonic mean identity. Bayesian Statistics 8: 1-45.

[11] Shachter RD and Kenley R (1989) Gaussian influence diagrams. Management Science, 35: 527-550.

[12] Yi N, Yandell BS, Churchill GA, Allison DB, Eisen EJ, Pomp D (2005) Bayesian model selection for genome-wide epistatic quantitative trait loci analysis. Genetics 170: 1333-1344.

[13] Winrow CJ, Williams DL, Kasarskis A, Millstein J, Laposky AD, Yang HS, Mrazek K, Zhou L, Owens JR, Radicki D, Preuss F, Schadt EE, Shimomura K, Vitaterna MH, Zhang C, Koblan KS, Renger JJ, TUREK FW (2009) Uncovering the genetic landscape for multiple sleep-wake traits. PLoS ONE 4: e5161.

E. Chaibub Neto and B. S. Yandell

Department of Statistics

UNIVERSITY OF WISCONSIN - MADISON

1300 University Avenue

Madison, Wisconsin 53706 USA

E-MAIL: chaibub@stat.wisc.edu

byandell@wisc.edu
M. P. Keller and A. D. Attie Department of Biochemistry

University OF Wisconsin - MADison

433 Babcock Drive

MADISON, Wisconsin 53706 USA

E-MAIL: mkeller3@wisc.edu adattie@wisc.edu 